ARTICLE

Received 10 Jul 2015 | Accepted 17 Feb 2016 | Published 30 Mar 2016

DOI: $10.1038 /$ ncomms11063 OPEN

\title{
Essential role of the TFIID subunit TAF4 in murine embryogenesis and embryonic stem cell differentiation
}

Diana Langer ${ }^{1}$, Igor Martianov ${ }^{1, \star}$, Daniel Alpern ${ }^{1,2, \star}$, Muriel Rhinn ${ }^{3, \star}$, Céline Keime ${ }^{1}$, Pascal Dollé ${ }^{3}$ Gabrielle Mengus $^{1}$ \& Irwin Davidson ${ }^{1}$

TAF4 (TATA-binding protein-associated factor 4) and its paralogue TAF4b are components of the TFIID core module. We inactivated the murine Taf4a gene to address Taf 4 function during embryogenesis. Here we show that Taf4a-/- embryos survive until E9.5 where primary germ layers and many embryonic structures are identified showing Taf4 is dispensable for their specification. In contrast, Taf4 is required for correct patterning of the trunk and anterior structures, ventral morphogenesis and proper heart positioning. Overlapping expression of Taf $4 a$ and Taf $4 b$ during embryogenesis suggests their redundancy at early stages. In agreement with this, Taf4a- /- embryonic stem cells (ESCs) are viable and comprise Taf4b-containing TFIID. Nevertheless, Taf $4 a^{-/-}$ESCs do not complete differentiation into glutamatergic neurons and cardiomyocytes in vitro due to impaired preinitiation complex formation at the promoters of critical differentiation genes. We define an essential role of a core TFIID TAF in differentiation events during mammalian embryogenesis.

\footnotetext{
${ }^{1}$ Department of Functional Genomics and Cancer, Institut de Génétique et de Biologie Moléculaire et Cellulaire, CNRS/INSERM/ULP, 1 Rue Laurent Fries, 67404 IIlkirch, France. ${ }^{2}$ L'École polytechnique fédérale de Lausanne, Route Cantonale, 1015 Lausanne, Switzerland. ${ }^{3}$ Department of Development and Stem Cells, Institut de Génétique et de Biologie Moléculaire et Cellulaire, CNRS/INSERM/ULP, 1 Rue Laurent Fries, 67404 IIIkirch, France. ${ }^{\star}$ These authors contributed equally to this work. Correspondence and requests for materials should be addressed to I.D. (email: irwin@igbmc.fr).
} 
F ormation of the RNA polymerase II (Pol II) preinitiation complex (PIC) requires a set of general transcription factors among which is TFIID, a multiprotein complex composed of the TATA-binding protein (TBP) and a set of TBP-associated factors $(\mathrm{TAFs})^{1}$. TFIID assembles from a 'core complex' comprising TAF4, TAF5, TAF6, TAF9 and TAF12, followed by TAF8-TAF10, and then association with a second module comprising TBP, TAF1, TAF2 and TAF7 (refs 2,3). In mammals, core TAFs are almost ubiquitously expressed, although cell-specifically expressed paralogues of TBP and a subset of TAFs have been described and their functions defined by mouse knockouts. Taf7l plays a critical role in male germ cell development and in adipocytes ${ }^{4-6}, \mathrm{Taf} 4 \mathrm{~b}$ is essential for male and female fertility ${ }^{7,8}$ and Taf9b regulates neuronal gene expression ${ }^{9}$. Trf2 and Trf3, the two TBP paralogues, play essential roles in the male and female germ lines, respectively ${ }^{10,11}$.

TAF4 forms a histone fold heterodimer with TAF12 (refs 12-14) that associates with the TAF6-TAF9 heterodimer and TAF5 to form the TFIID core module. TAF4 is crucial for the structural integrity of the core module and TFIID ${ }^{1,15}$. Its paralogue TAF4B also heterodimerizes with TAF12 and integrates into TFIID, thus maintaining TFIID integrity in the absence of TAF4 (ref. 16). While we have used somatic inactivation to address the function of murine Taf4 in mouse embryonic fibroblasts (MEFs), the adult murine epidermis or neonatal liver ${ }^{16-19}$, its role in embryogenesis is unknown.

Here we characterise Taf $4 a^{-1-}$ (gene encoding Taf4) embryos showing they survive until E9.5, but exhibit severe growth retardation and specific defects in anterior and ventral patterning and morphogenesis. We also show that Taf4a-1- embryonic stem cells (ESCs) are viable, but are unable to properly differentiate into glutamatergic neurons and cardiomyocytes in vitro due to impaired PIC formation at the promoters of critical differentiation genes. Thus, while Taf $4 \mathrm{~b}$ can compensate for the absence of Taf4 during the early stages of embryogenesis and in ESCs, Taf4 plays specific roles in differentiation in vivo and in vitro.

\section{Results}

Mid-gestation embryonic lethality of Taf $4 a^{-/-}$embryos. Mice with floxed and null Taf4a alleles ${ }^{16}$ (Taf4 $a^{\text {lox } / ~}$ and Taf $4 a^{+1-}$ ) were viable, fertile and did not exhibit any apparent abnormalities. Breeding of Taf $4 a^{+1-}$ mice did not yield any Taf $a^{-1-}$ pups, indicating death in utero ${ }^{16}$. We, therefore, analysed embryogenesis at various developmental stages after crosses of Taf $4 a^{+l-}$ animals.

Taf $4 a^{-1-}$ embryos could be distinguished from Taf $4 a^{+1+}$ and Taf $4 a^{+1-}$ embryos by their smaller overall size at E6.5 (Fig. 1a-c). By E7.5 mutant embryos displayed a more compact appearance (Fig. 1d-g), and by E8.5, a shortened body axis and under-developed head and trunk regions (Fig. 1h-k). Between E8.5 and E9.5, mutant embryos showed virtually no increase in overall size, but developmental processes continued with for example formation of a primitive heart. Nevertheless, the overall morphology of Taf4a- ${ }^{-}$- embryos showed that their development did not proceed much beyond that of E8.5 wild-type (WT) embryos (Fig. 1k-m and see below). At E9.5, mutant embryos were severely growth retarded and subsequently resorbed (Fig. 11-o). Genotyping indicated that Taf $4 a^{-l-}$ embryos were present in approximately Mendelian ratios until E9.5 after which time they died and were longer detected (Supplementary Table 1).

Histology analysis showed that WT E7.5 embryos had completed division of the proamniotic cavity into the exocoelomic and the amniotic cavities, separated by the amniotic membrane (Fig. 1r), whereas in Taf $4 a^{-1-}$ embryos this compartmentalization had not
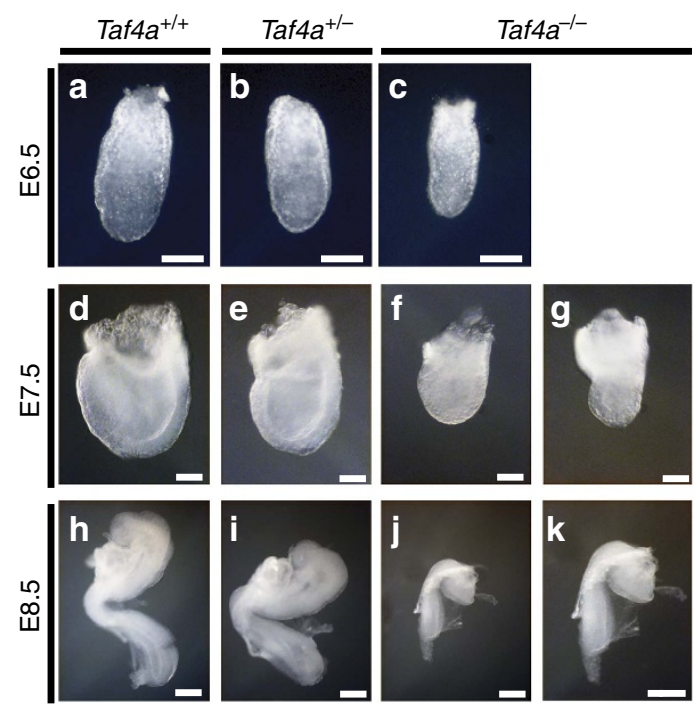

$\operatorname{Taf}_{4} \mathrm{a}^{+/+}$
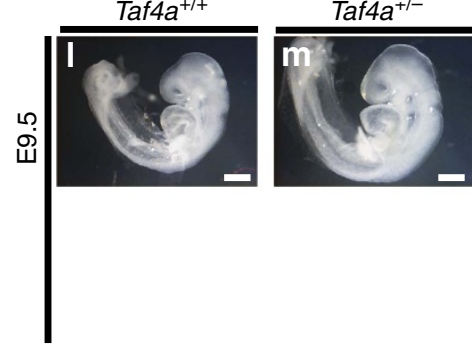

Taf4a $a^{-1-}$
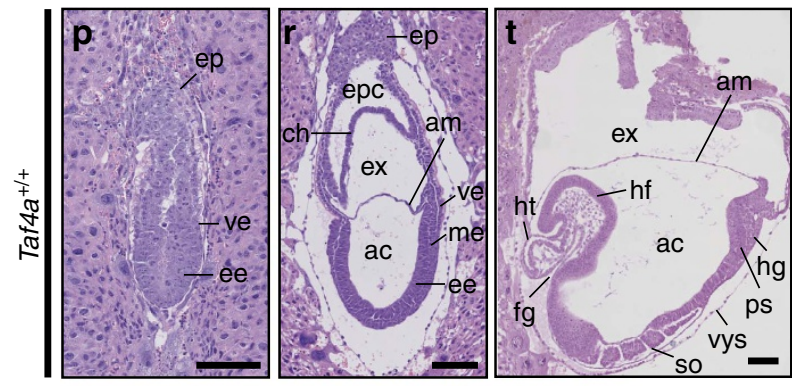

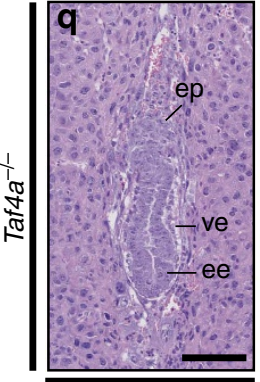

E6.5

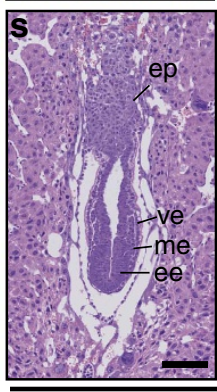

E7.5

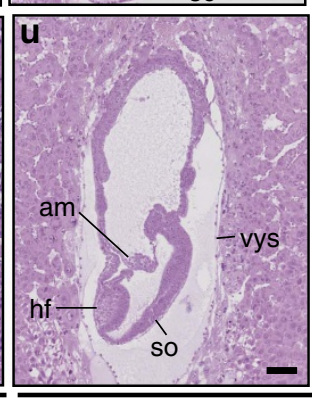

E8.5
Figure 1 | Taf4 is required during mid-gestation. (a-c) E6.5 stage embryos of the indicated genotypes. (d-g). E7.5-stage embryos of the indicated genotypes, $\mathbf{g}$ shows a blow up of $\mathbf{f}$. Scale bar, $100 \mu \mathrm{m}$. (h-k). E8.5-stage embryos of the indicated genotypes, $\mathbf{k}$ shows a blow up of $\mathbf{j}$. (I-o) E9.5stage embryos of the indicated genotypes, $\mathbf{o}$ shows a blow up of $\mathbf{n}$. Scale bar, $200 \mu \mathrm{m}$. (p-u) Haematoxylin and eosin-stained sections through WT or mutant embryos at the indicated stages. Scale bar, $100 \mu \mathrm{m}$. ep, ectoplacental cone; ve, visceral endoderm; ee, embryonic ectoderm; me, mesoderm;, epc, ectoplacental cavity; ch, chorion; ex, exocoelomic cavity; am, amnion; ac, amniotic cavity; hf, headfold; ht, heart; fg, foregut; hg, hindgut; so, somites; vys, visceral yolk sac; ps, primitive streak. 

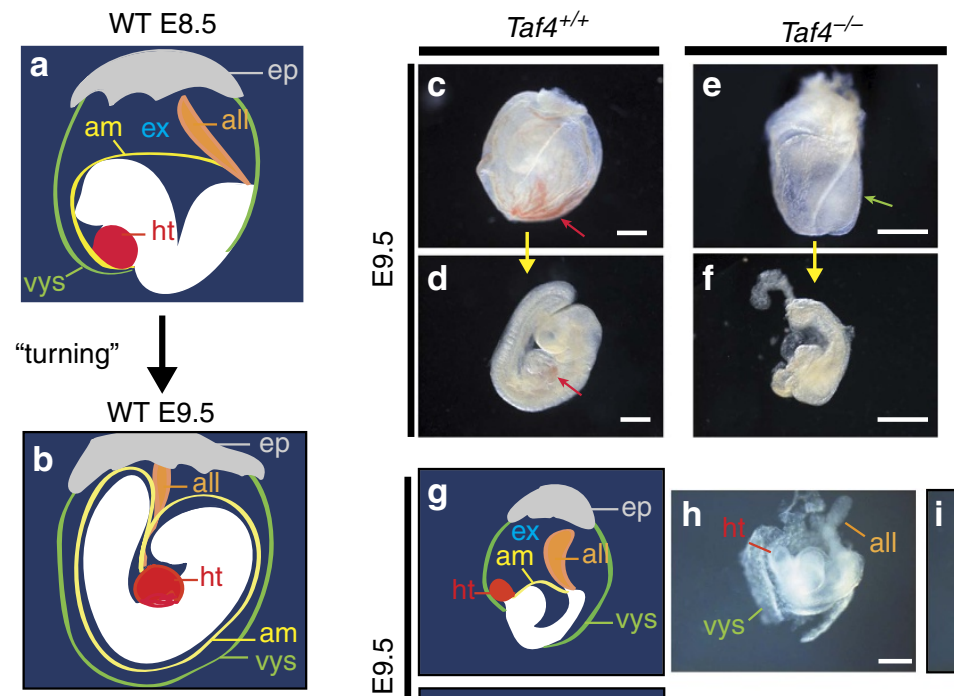

Final fetal position
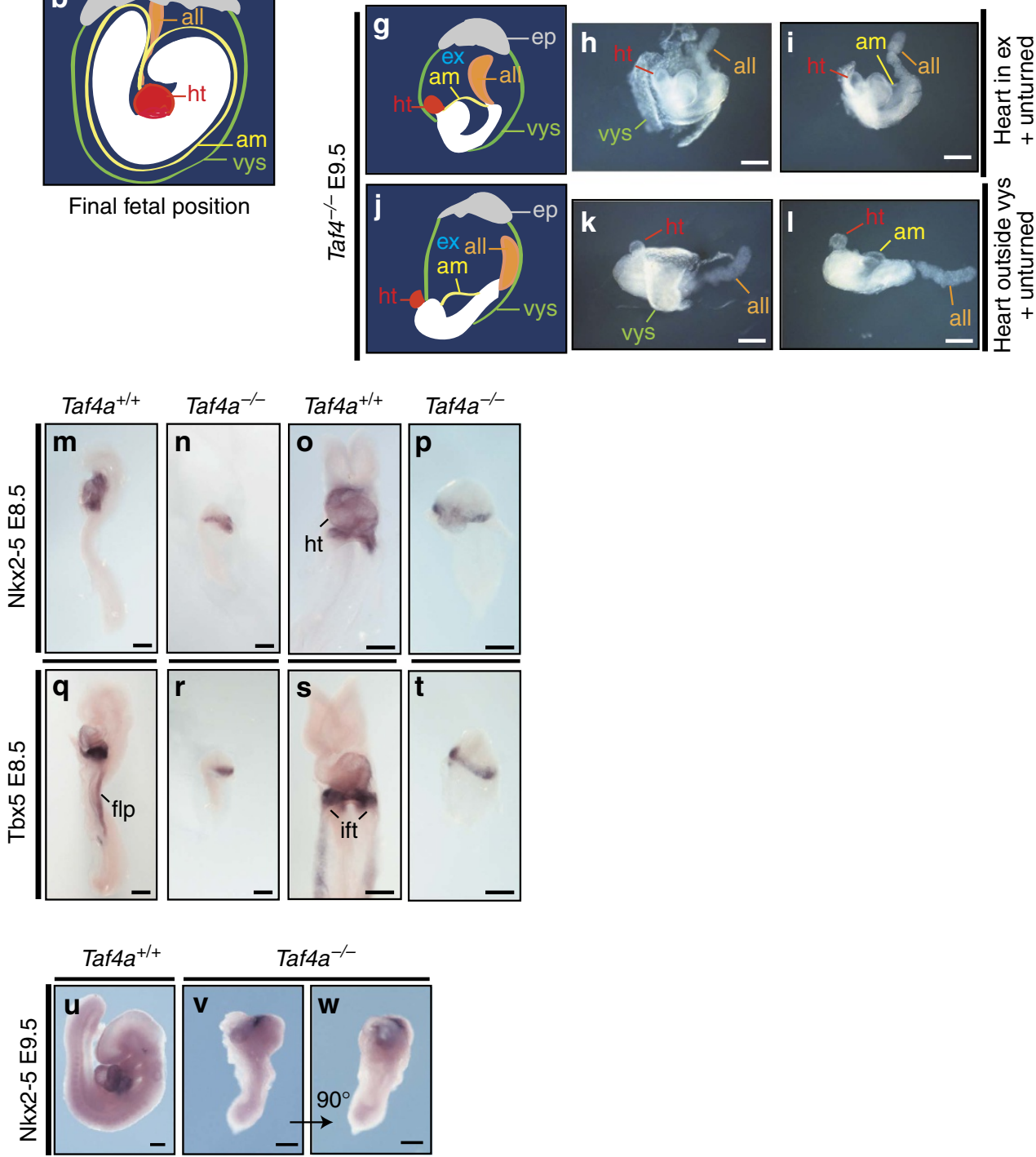

Figure 2 | Taf4 is required for correct positioning of head and heart. (a,b). Schematic representations illustrate normal development at E8.5 and E9.5 highlighting positions of head and heart during embryo turning. (c-f) WT and mutant embryos at E9.5 before and after dissection from the yolk sac highlighting the blood-filled yolk sac vascular plexus in control embryos (red arrow in c) and the embryo protruding partially out of the yolk sac (green arrow in e). WT embryos show a strong accumulation of blood in the atrium of the heart (red arrow in d), which was absent in Taf4a-/- embryos (f). (g-i) Schematic illustration of observed mutant phenotypes at E9.5. All mutant embryos fail to undergo turning at E9.5 and fusion of the allantois to the chorion. In addition to this, mutant embryos develop heart structures either in the exocoelomic cavity (h-i) or outside of the visceral yolk sac (k-I).

$(\mathbf{m}-\mathbf{w})$ In situ hybridization of wild-type and Taf4a-/- embryos for the cardiac transcription factors Nkx2-5 at E8.5 (m-p) or Tbx5 at E8.5 (q-t) and E9.5 (u-w). ep, ectoplacental cone; all, allantois; ht, heart; flp, forelimb precursors; ift, inflow tract. Scale bar, $500 \mu \mathrm{m}(\mathbf{c}-\mathbf{f}), 300 \mu \mathrm{m}(\mathbf{h}-\mathbf{i}), 200 \mu \mathrm{m}(\mathbf{m}-\mathbf{w})$.

structures and showed additional staining in the prospective forelimb field (Fig. 2q,s). In mutant embryos, Tbx5 showed a crescent-like expression domain, with no expression in the prospective forelimb (Fig. 2r,t). These crescent-like domains in the E8.5 mutant embryos closely resembled the expression pattern of heart-specific markers in the cardiac crescent of E7.5 WT embryos. Importantly however, histology analyses and in situ hybridization indicated mis-localization of the primitive heart 

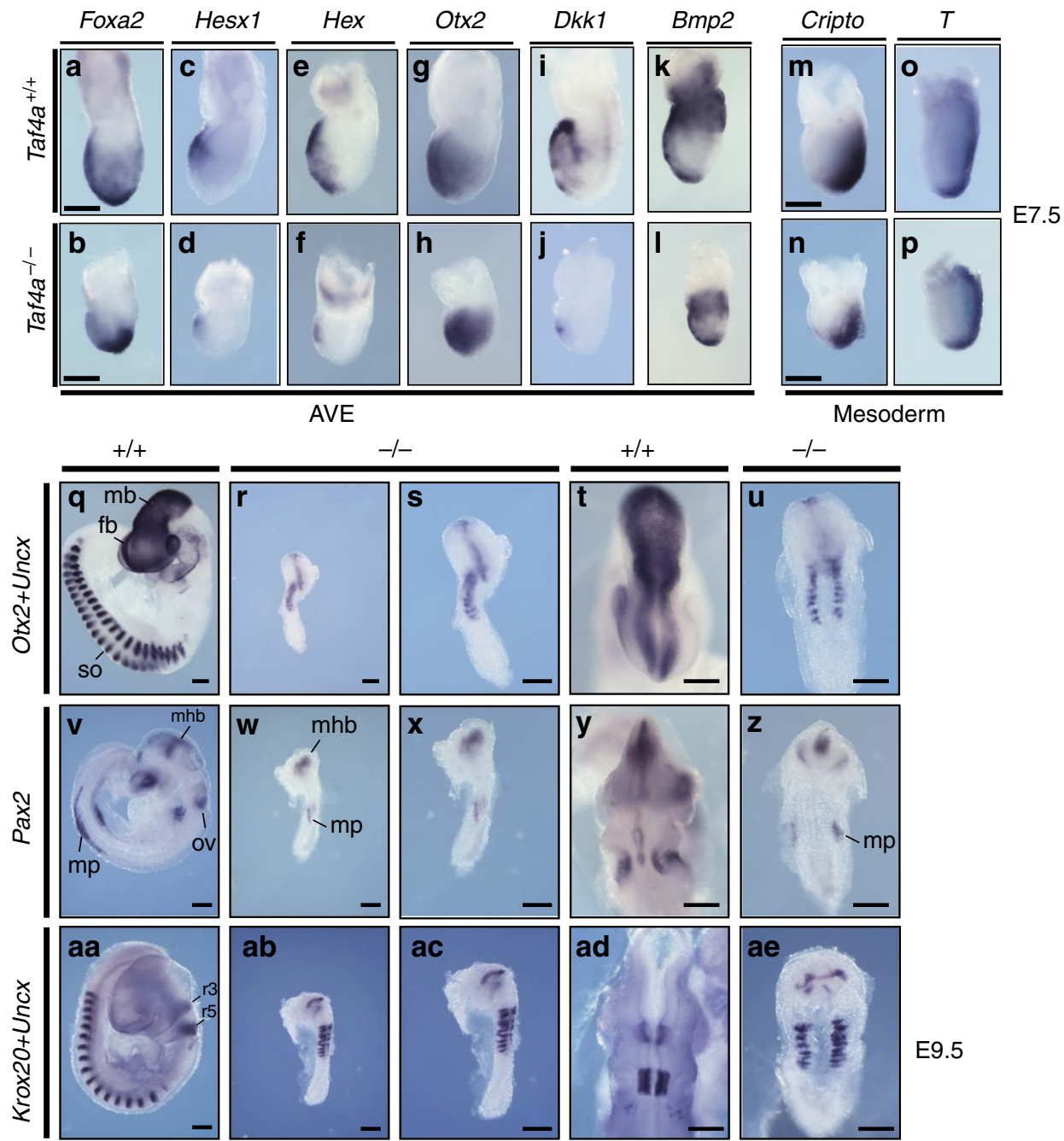

E9.5
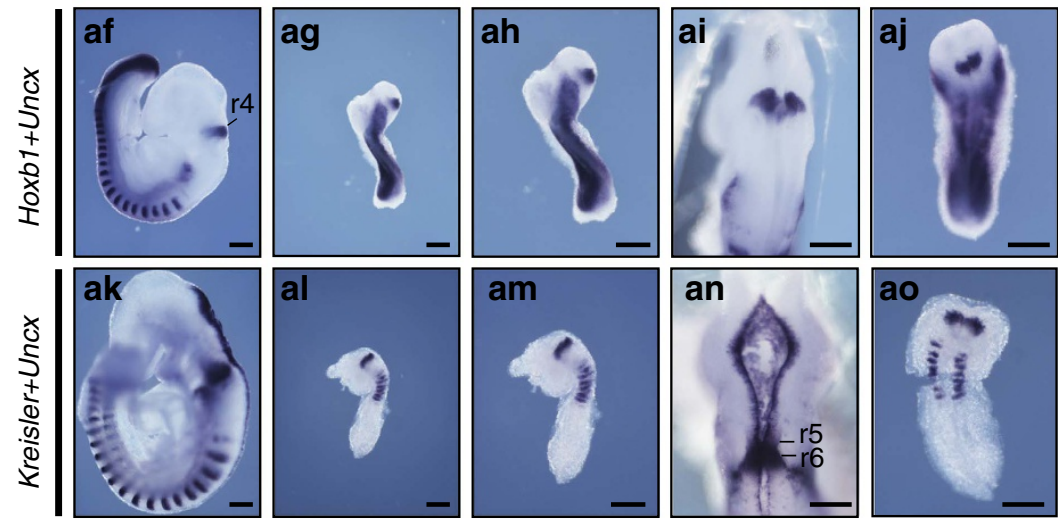

Figure 3 | Abnormal patterning of Taf4a- /- embryos. (a-o) (upper row). In situ hybridization with the indicated probes for anterior visceral endoderm (AVE) and mesoderm markers on E7.5 WT embryos. (b-p) (lower row). In situ hybridization with the indicated probes on E7.5 Taf4-/- embryos. (q-ao). In situ hybridization with neuronal markers in E9.5 WT or Taf4 ${ }^{-/-}$embryos (lateral views (q-ak); ventral view R dorsal views T-AN) and (lateral views RP$\mathrm{AL}$, higher magnifications of same embryos s-am; ventral view $\mathrm{U}$; dorsal views $\mathbf{z}$-ao). Scale bar, $200 \mu \mathrm{m}$ in all panels.

structures anterior to the headfolds rather than in the ventral position in keeping with the absence of turning of the mutant embryos. This was highlighted by in situ hybridization with Nkx2-5 at E9.5 labelling the developing heart structure at the anterior pole of the embryo next to the headfolds rather than in a ventral position as in WT (Fig. 2u-w). Taf $4 a^{-1-}$ embryos therefore display defective cardiac development that, together with lack of chorio-allantoic fusion and defective yolk sac and intra-embryonic vascularization, likely accounts for embryonic death at mid-gestation.

Abnormal anterior patterning of $T a f 4 a^{-/-}$embryos. We next used specific markers to investigate defective anterior development of Taf $4 a^{-1}$ embryos, The anterior visceral endoderm (AVE) regulates initial steps of head, heart and foregut 
positioning. Defective expression of AVE markers leads to severe anterior truncation phenotypes ${ }^{24}$. Expression domains of several AVE markers in E7.5 Taf4a-1- embryos were either reduced (Hesx1, Hex and Dkk1) or showed retarded formation (Foxa2, Otx2, Fig. 3a-1). In contrast, expression domains of mesodermal markers Brachyury (T) and Cripto were comparable in WT and mutant embryos (Fig. $3 \mathrm{~m}-\mathrm{p}$ ).

To investigate differentiation of specific anterior structures, we studied expression of forebrain, midbrain and hindbrain markers. Otx2 is required for induction and maintenance of the anterior neural plate $e^{25,26}$ and specification of forebrain and midbrain regions. The Otx 2 expression domain was diminished in E9.5 Taf $4 a^{-1-}$ mutants, particularly the forebrain was strongly reduced (Fig. 3q-u). Pax2 was expressed in the developing midbrain-hindbrain region, the otic vesicle, and the mesonephros in WT embryos, whereas in mutant embryos midbrain-hindbrain staining was reduced, and the presence of nephrogenic precursor cells was observed. In contrast, no staining of presumptive otic vesicle structures was observed (Fig. 3v-z).

In WT E9.5 embryos, Krox 20 marked rhombomeres 3 and 5. In mutant embryos, labelled structures were thinner and
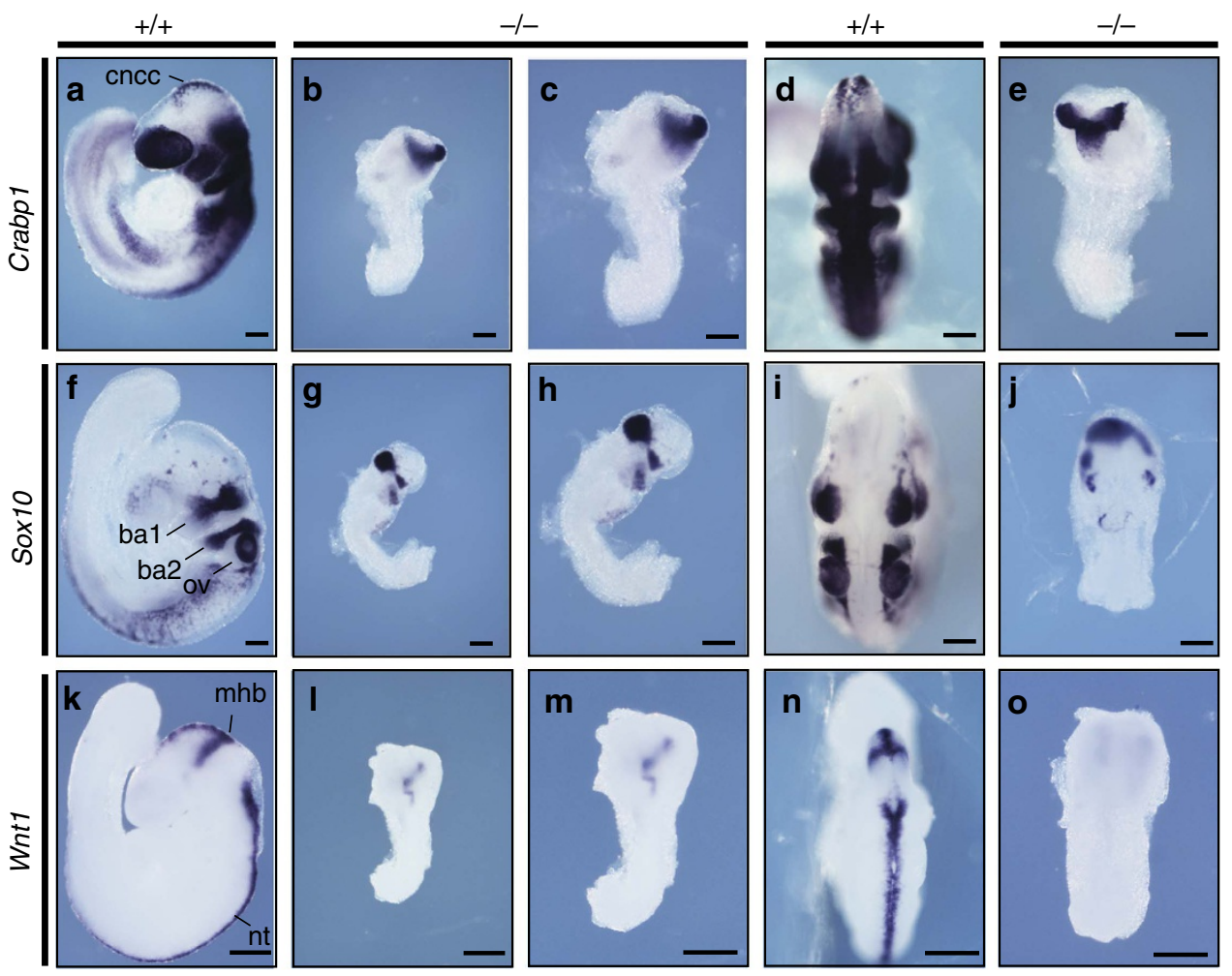

Pou5f1

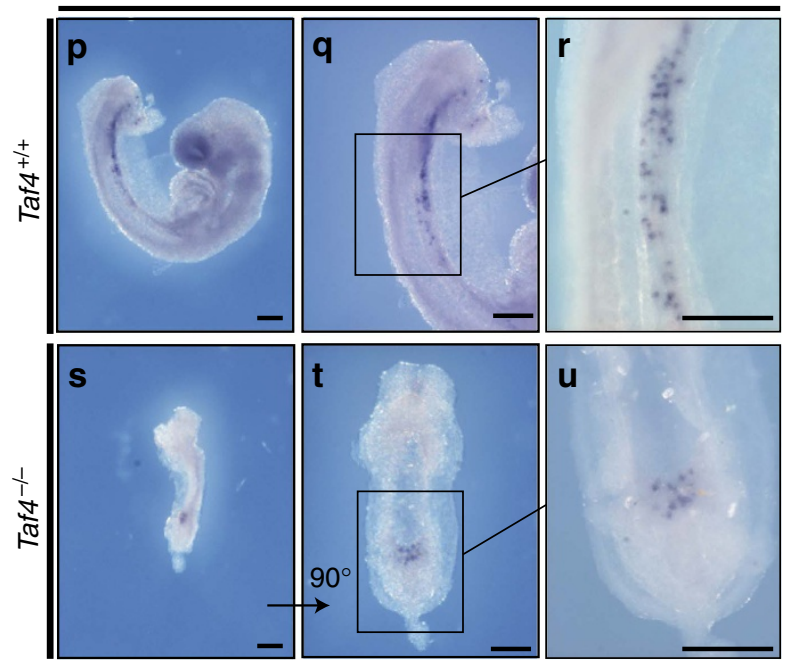

Figure 4 | Neural crest cell markers and primordial germ cells in Taf4 mutant embryos. (a-o) In situ hybridizations for neural crest markers Sox10, Crabp1

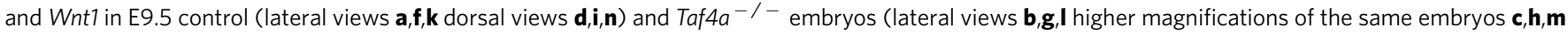
dorsal views E, J, O ). (p-u). In situ hybridizations at E9.5 with Pou5f1 (Oct4) as a marker for primordial germ cells (PGCs). ba1, first branchial arch; ba2, second branchial arch; ov, otic vesicle; cncc, cranial neural crest cells; mhb, midbrain-hindbrain boundary; nt, neural tube. Scale bar, $200 \mu \mathrm{m}$ in all panels. 
disorganized with rhombomere 5 more affected than rhombomere 3 (Fig. 3aa-ae). Hoxb1, a marker for rhombomere 4, was clearly detected in mutant embryos (Fig. 3af-aj), whereas Mafb (Kreisler), expressed in rhombomeres 5 and 6 in WT embryos (Fig. 3ak,an), stained only a single stripe in Taf $4 a^{-1-}$ mutants (Fig. 3al,am,ao), which in some instances, was thinner than the Hoxb1-positive stripe (compare Fig. 3ag,al). Thus, although the anterior region of mutant embryos was severely atrophied, this did not reflect a complete lack of forebrain/midbrain/hindbrain specification, all of which were present albeit both reduced in size and disorganized. Uncx staining showed fewer (typically 8-11) and irregularly shaped somites in E9.5 Taf $4 a^{-/-}$mutants.

The above analyses revealed an immediate proximity of the hindbrain and somite markers $(U n c x)$ and a reduction or absence of the hindbrain posterior to rhombomere 5 along with a significant portion of the trunk. We analysed the anteriorposterior axis and gut development in mutant embryos. Foxa2 and Shh labelled the notochord and developing hindgut in E9.5 WT embryos, but only one structure in mutant embryos (Supplementary Fig. 3B). Brachyury (T) staining indicated the labelled structure was the notochord showing interruption at one or several places, perhaps due to local apoptosis or tissue distortion (Supplementary Fig. 3C). The notochord showed stronger anterior staining by Brachyury and Foxa2 indicating defective elongation along the anterior-posterior axis. Nevertheless, mutant embryos maintained strong Brachyury staining in their posterior region, which was proportionally larger than in WT embryos at E8.5 and E9.5. This could indicate a defect in specification and differentiation of axial mesendodermal structures. Taf $4 a^{-1-}$ embryos therefore fail to develop a detectable gut tube consistent with histology analysis that showed lack of a foregut pocket E8.5 (Fig. 1u) and their shortened notochord is consistent with the absence of an elongated trunk region.

Reduced neural crest domain in Taf $4 \boldsymbol{a}^{-/-}$embryos. Having defined defective anterior and trunk development, we next investigated neural crest cells (NCCs) normally induced at the midbrain level and as a wave extending towards the tail region. Defects in NCCs may contribute to the defects observed in the cephalic and trunk region of the Taf $4 a^{-1-}$ embryos.

In E9.5 WT embryos, Crabp1 labelled the neural tube and NCCs originating in the posterior hindbrain and anterior trunk regions that give rise to cranial NCCs migrating into the branchial arches and posterior regions (Fig. 4a,d). A severely reduced Crabp1 expression domain was seen in mutant embryos, indicating they were completely devoid of migrating NCCs in both the cranial and posterior regions (Fig. $4 b, c, e)$. The residual $y$-shaped expression domain may correspond to part of the neural tube and primitive branchial arch 1. Similarly, Sox10, a marker with more restricted expression domains in the otic vesicle and the NCCs migrating into the branchial arches (Fig. 4f,i), also showed severely reduced expression in the mutant embryo and no migrating NCCs were observed (Fig. 4g,h,j). Furthermore, Wnt1 expression was absent from the spinal cord and NCCs (Fig. 4k-o). Taf $4 a^{-1-}$ embryos therefore lack a large portion of the NCCs migrating towards craniofacial structures and more posterior trunk regions.

Taf4 is dispensible for primordial germ cell generation. Given the role of Taf4 in reprogramming (ref. 27 and see below), we specifically investigated the presence of primordial germ cells (PGCs) in Taf4a-I- embryos. PGCs colonize the developing gonads by migration giving rise to oocytes and spermatocytes in adults $^{28}$. PGCs can be identified at around E7.25 (refs 29,30) and begin to migrate through the primitive streak to the developing hindgut, and by $\approx \mathrm{E} 9.5$ towards the genital ridges. Mutant embryos lack a properly developed hindgut mesentery at E9.5. We investigated whether mutants were able to initiate PGC development. At E7.5 Pou5f1 (Oct4) is exclusively expressed by the newly formed PGCs, and expression persists as they migrate and proliferate. We readily detected Pou5f1-expressing PGCs in the WT hindgut mesentery at E9.5 (Fig. 4p-r). In mutant embryos, Pou5f1-expressing PGCs could be observed, but were around 10-fold less numerous than in WT (Fig. $4 \mathrm{~s}-\mathrm{u}$ ). These PGCs accumulated at the presumptive entry site into the hindgut lacking in mutant embryos. Thus, PCG specification takes place in absence of Taf4, but they cannot migrate and pursue their proliferation due to the absence of the hindgut.

Taf $4 a^{-/-}$ESCs are viable and comprise TFIID containing Taf $4 \mathrm{~b}$. The lethality, reduced size and pleiotropic defects of the Taf $4 a^{-1-}$ embryos preclude biochemical and functional analysis of specific differentiation events. Nevertheless, as death occured at mid-gestation, it should be possible to derive Taf $4 a^{-1-}$ ESCs and use them to address the role of Taf4 in specific differentiation programmes. We isolated ESCs from blastocysts of Taf $4 a^{+1-}$ heterozygous crosses and established two homozygous null lines and two WT lines from these crosses. The absence of Taf 4 protein in Taf $4 a^{-1}$ ESCs was confirmed by immunostaining, while pluripotency markers Pou5f1, Nanog and Sox2 were normally expressed (Supplementary Fig. 4AC). WT and Taf $4 a^{-1-}$ ESCs showed comparable alkaline phosphatase staining, proliferation rates and cell cycle profiles (Supplementary Fig 4D-F). Comparative RNA sequencing (RNAseq) revealed only minor differences in gene expression. Around 40 genes were upregulated although only 12 showed more than a threefold change, whereas 166 genes were downregulated (Supplementary Fig. 4G) including Noggin and several genes associated with Wnt signalling. However, their altered expression did not elicit detectable changes in cell physiology and no change in pluripotency gene expression was observed. Together these data show that Taf $4 a^{-1}$ ESCs are viable and comparable in most essential parameters to WT ESCs.

To characterise ESC TFIID and to compare it with human ESCs and MEFs, we performed western blot analysis. WT mouse ESCs express Tbp and all tested Tafs, including Taf $4 b$. For equivalent amounts of protein extract, expression of many Tafs was higher in ESCs than in MEFs (Fig. 5a), consistent with previous reports ${ }^{27}$. In two human ESC lines, no expression of TAF1, TAF4 and TAF5 was seen, although TAF4b was detected. These observations confirm previous reports of noncanonical TFIID composition in human $\mathrm{ESCs}^{31}$, and highlight fundamental differences in the biochemistry of mouse and human ESCs.

Comparison of WT and Taf $4 a^{-1}$ ESCs indicated comparable expression of most TAFs. Tbp was immunoprecipitated (IP) from both lines and equivalent amounts of Tbp loaded on immunoblots (Supplementary Fig. 5). Taf4b was co-precipitated with Tbp in both the presence and absence of Taf 4 indicating that Taf $4 \mathrm{~b}$ maintained TFIID integrity in Taf $4 a^{-1}$ ESCs. Nevertheless, reduced levels of several TAFs, with respect to Tbp, were observed in the Tbp IP from the Taf $4 a^{-/}$ESCs suggesting that Taf $4 \mathrm{~b}$ expression was not sufficient to maintain an overall TFIID level comparable to WT ESCs.

We then performed gel filtration and analysed the presence of Tbp and TAFs in the high-molecular mass fractions. Tbp and TAFs coeluted in a high molecular mass complex of around $2 \mathrm{mDa}$ in WT extracts (Fig. 5b). Taf4b was also present in these fractions, although a subfraction eluted in a lower molecular mass complex. In Taf $4 a^{-1-}$ extracts, Taf4b was present along with 
a

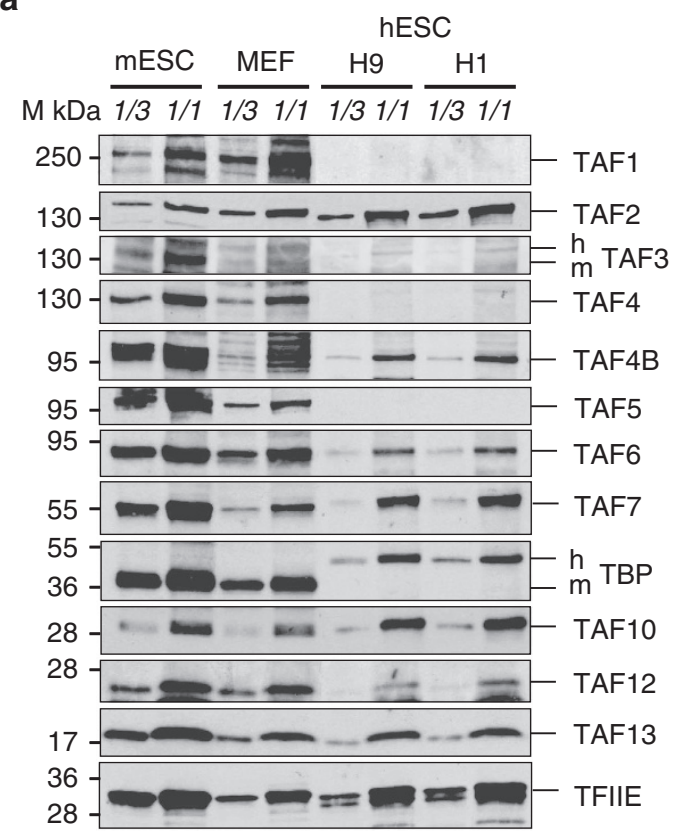

C

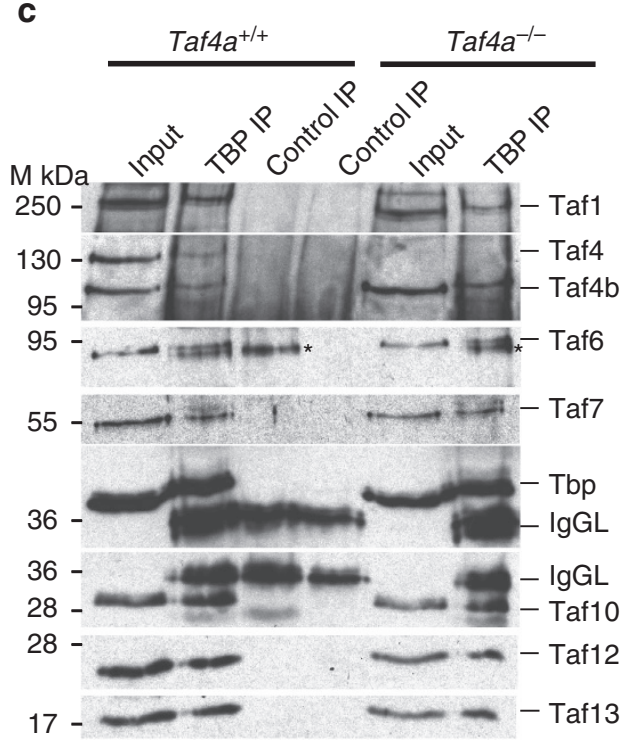

b

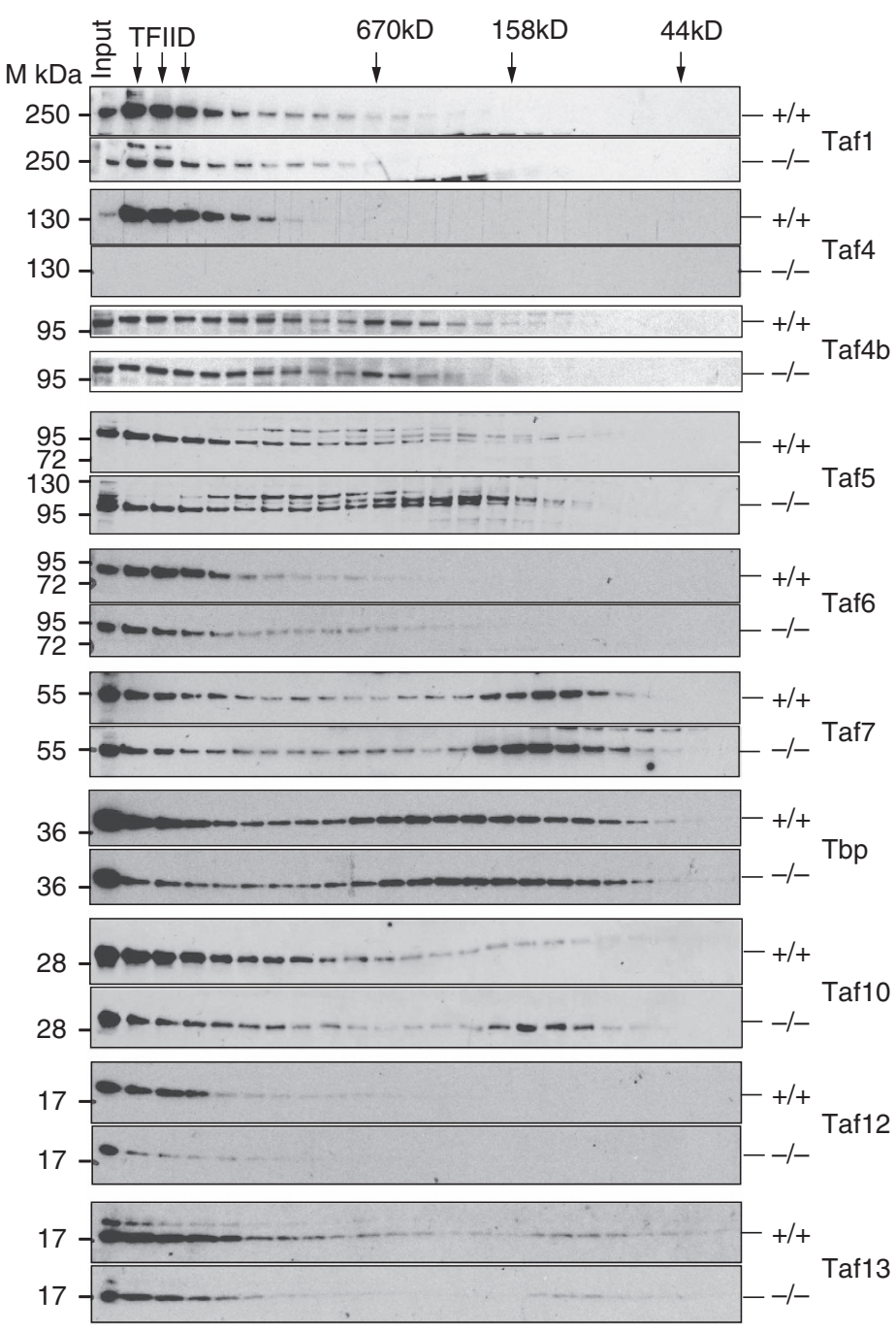

Figure 5 | Biochemical analysis of TFIID in Taf4a ${ }^{-/}$ES cells. (a). Immunoblots showing expression of TFIID subunits in ES cells, MEFs and two human ES cell lines, H1 and H9. (b). Immmunoblots on gel filtration fractions of nuclear extracts from WT and mutant ES cells. (c). Immunoblots of Tbp IPs from the pooled TFIID fractions indicated by arrows in $\mathbf{b}$. * indicates a non-specific band migrating just below Taf6.

Tbp and other tested TAFs in high-molecular mass fractions, although Tbp and many TAFs were present in reduced amounts in particular Taf7 and Taf10 that were increased in lower molecular weight fractions.

To investigate whether the high-molecular mass fractions correspond to TFIID, the indicated fractions were pooled and subjected to Tbp IP. In fractions from WT and mutant ESCs, Tafs $1,4 \mathrm{~b}, 6,7,10,12$ and 13 were co-precipitated with Tbp showing formation of a TFIID complex in both cell types (Fig. 5c). In fractions from WT cells, both Taf4 and Taf4b were precipitated, while in the fractions from mutant cells only Taf4b was present. Taf4b therefore integrates in TFIID in Taf $4 a^{-1-}$ ESCs, but these cells contain lower overall levels of TFIID as the presence of Taf4b cannot quantitatively compensate Taf4 loss.
To further verify potential redundancy between Taf4 and Taf4b, we transfected WT and Taf4a ${ }^{-1}$ ESCs with control small interfering RNA (siRNA) and siRNAs directed against Taf4b. After control siRNA and siTaf4b silencing in WT ESCs and replating the transfected cells, comparable numbers of alkaline phosphatase staining colonies were observed expressing comparable levels of Nanog, showing that Taf4b knockdown did not compromise cell viability (Supplementary Fig. 6A-C). In contrast, Taf $4 \mathrm{~b}$ silencing in Taf $4 \mathrm{a}^{-/}$ESCs led to a strong reduction in the number of colonies and surviving cells showed normal Taf4b and Nanog expression. Hence, Taf4b silencing in Taf4a $a^{-1-}$ ESCs strongly compromised cell viability and only cells that had escaped transfection formed colonies. These results indicate that ESCs lacking either Taf 4 of Taf $4 \mathrm{~b}$ were viable. In 
contrast, loss of both proteins through genetic deletion of Taf4 and subsequent Taf $4 \mathrm{~b}$ silencing resulted in a loss of viability. Taf4 and Taf $4 \mathrm{~b}$ are therefore redundant in terms of assuring cell ESC viability.

Defective neuronal differentiation of $\mathrm{Taf}_{4} \mathrm{a}^{-/-}$ES cells. Taf4a ${ }^{-1}$ ESCs were grown as embryoid bodies (EBs) to determine their spontaneous differentiation capacity over a period of 14 days. Taf $4 a^{-1-}$ EBs initially appeared indistinguishable from WT EBs, but at later stages, gave rise to fewer cystic-like structures (Supplementary Fig. 7A) that resemble the developing yolk sac in vivo ${ }^{32}$. Hence, in vitro differentiated Taf4a $a^{-1}$ ES cells recapitulated to some extent the phenotype of the Taf4a ${ }^{-1-}$ embryos. Taf4 was expressed throughout WT EB differentiation, while Taf $4 b$ expression was strongly decreased, but Taf $4 b$ was maintained in the mutant cells (Supplementary Fig. 7B).

We investigated whether Taf $4 a^{-7-}$ ESCs could give rise to the three primary germ layers. Expression of the pluripotency markers Nanog and Dppa3 was reduced, but incompletely repressed compared with WT (Supplementary Fig. 7B). The early endoderm and mesoderm markers Gata4 and Gata6 were induced, whereas late endodermal lineage markers like Foxa2, and $A f p$ were strongly downregulated (Supplementary Fig. 7B). Mesendodermal markers Brachyury (T) and Goosecoid (Gsc) showed a strong upregulation in day 6 EBs before their expression returned to normal levels, indicating that Taf4 may regulate the fine-tuning of their expression. Expression of the early cardiac marker Nkx2-5 and its downstream target gene Anf was also reduced. Taf $4 a^{-1-}$ ESCs can therefore initiate differentiation into early mesoendodermal-type cells, whereas later stage differentiation markers were more strongly affected.

Expression of the early ectodermal marker Fgf5 was induced, but expression of later neuronal markers such as Pax6 and Mtap2 and $D c x$ was strongly reduced (Supplementary Fig. 7B). To investigate the role of Taf4 in neuronal differentiation, we differentiated WT and Taf4a-l- ESCs into glutamatergic neurons by treating EBs with all-trans retinoic acid (RA) (Supplementary Fig. 8A, refs 33,34). Expression of the vesicular glutamate transporter Vglut2 as well as Taf4 was observed in such neurons cultured for 12 days (Supplementary Fig. 8B).

Dissociated RA-treated WT EBs differentiated into neuronal precursors shortly after plating and adopted neuronal morphology with abundant axons and prominent Tubb3 staining after 2 days (Fig. 6a,b). In contrast, mutant EBs failed to generate neuronal precursors with an axonal network and most cells died within a day. After 1-2 days, the few remaining cells appeared flattened and enlarged. Western blot showed only weak Tubb3 expression in mutant cells, but also revealed defective repression of Pou5f1 and Nanog whose expression was strongly repressed after differentiation of WT EBs (Fig. 6c). Taf4 is therefore essential for RA-induced neuronal differentiation and for pluripotency gene repression.

Immunoblot analysis showed a mild overall reduction in Tbp and Taf expression by day 8 of WT EB differentiation (Fig. 6d). On differentiation of mutant EBs, the overall reduction in Taf levels was less marked with no decreased Taf $4 \mathrm{~b}$ expression, whereas Taf3 and Taf5 were reduced.

To demonstrate that defective differentiation was due to Taf4 loss, we generated Taf $4 a^{-1}$ ESCs stably re-expressing exogenous full-length Taf4 (Fig. 6e). WT ESCs isolated in parallel after transfection with control vector showed normal neuronal differentiation with an abundant Tubb3-stained axonal network, whereas the Taf4a $a^{-1-}$ EBs transfected with control vector showed no neuronal differentiation (Fig. 6f). Importantly, the rescue $\mathrm{Taf}_{4 a^{-1-}}$ line showed comparable differentiation to the
WT lines. Defective differentiation was therefore directly attributable to Taf4 loss and restored by its re-expression. We also attempted to rescue differentiation by the expression of Taf4 (372-1,083) and Taf4 (805-1,083); however, no stable clones could be isolated suggesting that expression of truncated Taf4 was toxic in these cells. The integrity of Taf4 is therefore required to rescue the differentiation defects.

We performed RNA-seq on two independent WT and mutant ESC lines before (EB4) and 24 (EB5) and 96h (EB8) after RA treatment. At EB4 585 deregulated genes were observed (354 down and 231 up, Fig. 7a). Similarly, at EB5, 255 upregulated and 287 downregulated genes were detected, but the highest number of deregulated genes was observed at EB8 (1,212 up and 1,008 down). Thus, Taf4b can replace Taf4 to maintain cellular housekeeping functions and pluripotency and to a limited extent the initial stages of differentiation, but Taf4 is required for full differentiation with progressively more severe alterations in gene expression as differentiation progressed (Fig. 7a).

At EB8, genes involved in neurogenesis are strongly downregulated, whereas many pluripotency genes are prominent in the upregulated class (Fig. 7b,c). Downregulated genes are highly associated with the brain, while upregulated genes are associated with ESCs and spermatogenesis reflecting the presence of stemlike genes expressed in PGCs and spermatogonia (Supplementary Fig. 8C). Clustering identified genes with distinct kinetics of expression in WT cells and altered expression in Taf4 $4 a^{-1-}$ cells. Three clusters show the most prominent changes. Genes in cluster 2 were upregulated when WT ES cells were grown as EBs, but were most strongly induced after RA treatment at EB5 and were highly enriched in brain and neurogenesis functions (Fig. 7d). In mutant ESCs, their activation was strongly reduced whereby expression at EB8 was comparable to that of WT EB4. Genes in cluster 3 were also strongly enriched in brain and neurogenesis, but were mainly activated between EB5 and EB8 and were strongly reduced in mutant cells. In contrast, genes in cluster 4 are ES-expressed and pluripotency genes downregulated in WT EBs, then switched off during their differentiation, whereas in Taf4 $4 a^{-1-}$ EBs, their expression persisted during differentiation.

Similar to Taf4a $a^{-1-}$ cells in vitro, expression of several neuronal genes was downregulated in Taf $4 a^{-1-}$ embryos showing that Taf4 is also required for their normal expression in vivo (Supplementary Fig. 8D). Expression of Pouffl, and Nanog was, however, repressed in Taf $4 a^{-1}-$ embryos, but that of other pluripotency associated genes like Zfp42 and Dppa5a remained elevated at E9.5.

The above data show that WT ESCs differentiated into neuronal precursors that switched off pluripotency gene expression, whereas Taf $4 a^{-1-}$ ESCs adopt a unique state with partially induced expression of differentiation genes, and partially repressed expression of pluripotency genes.

Defective PIC formation at neuronal genes. The above classification based on kinetics of gene expression also reflects different regulatory mechanisms as revealed by ChIP-seq. TFIIB, Pol II, $\mathrm{H} 3 \mathrm{~K} 4 \mathrm{me} 3$ and $\mathrm{H} 3 \mathrm{~K} 27 \mathrm{ac}$ were present at promoters of genes in cluster 1 in WT ESCs. Consistent with increased expression of these genes during differentiation, TFIIB, Pol II and H3K27ac were increased at EB8 (Fig. 7e). In contrast, lower occupancy was seen in Taf4a $a^{-1}$ ESCs and little change was observed at EB8 in accordance with their reduced activation. TFIIB, Pol II, H3K4me3 and low levels of H3K27ac were observed at cluster 2 promoters in WT and mutant ESCs and activation of these genes was associated with increased occupancy in WT EB8, but not in Taf4a $a^{-1-}$ EB8. Increased elongating Pol II was also observed in 


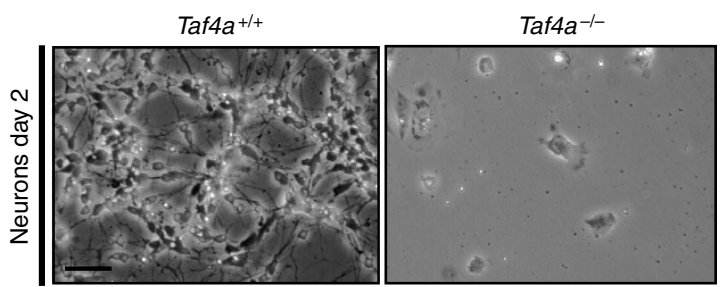

b

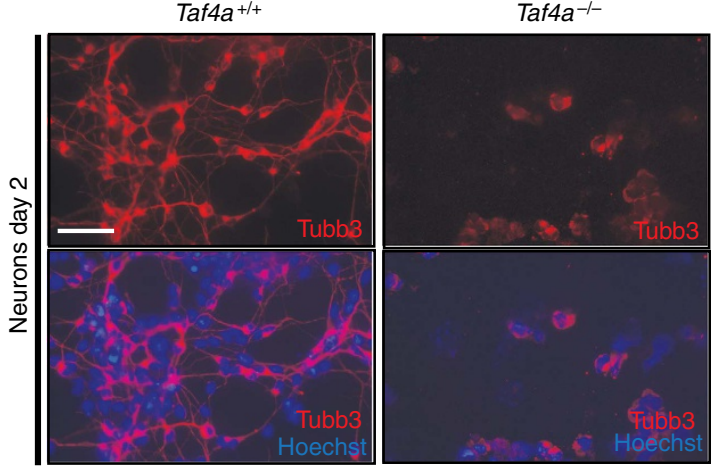

C
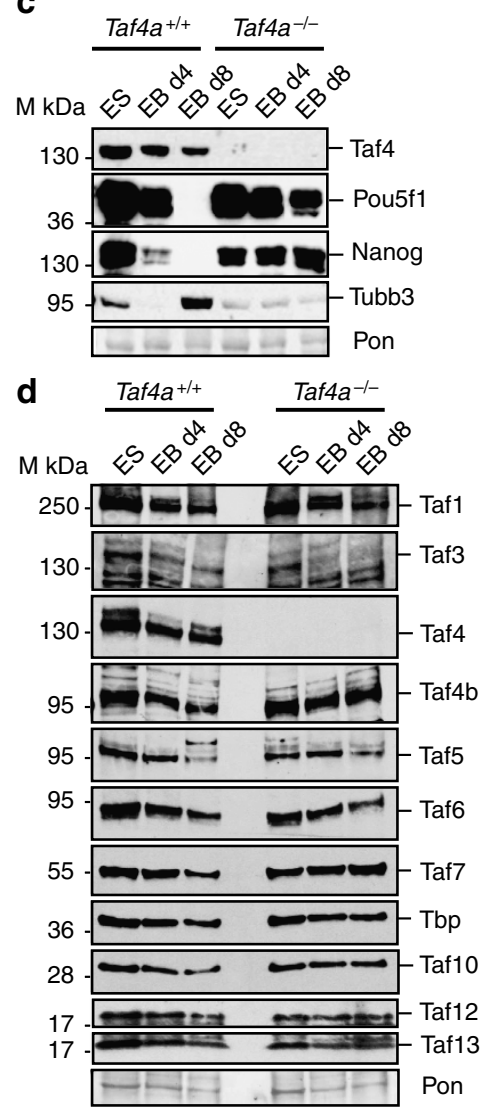

f

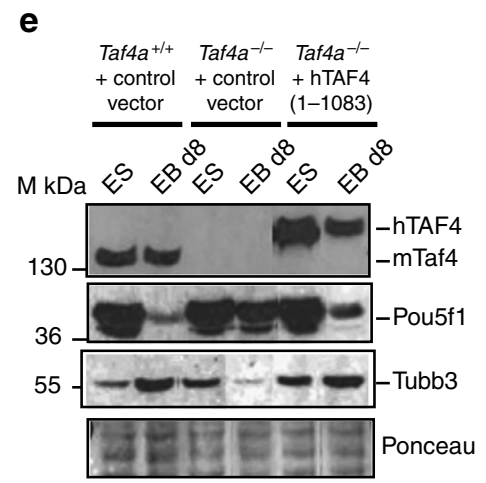

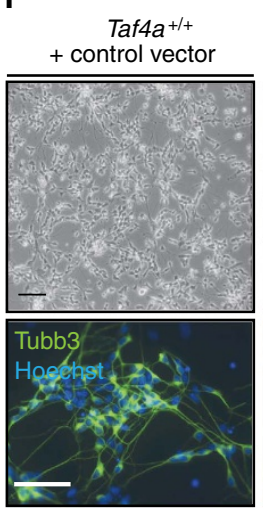

Taf4a-/+ control vector

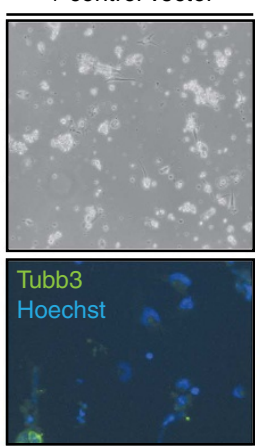

Taf4a-/+ hTAF4(1-1083)

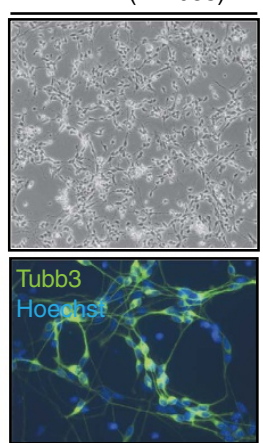

Figure 6 | Taf4 is required for RA-driven neuronal ES cell differentiation. (a) Phase-contrast microscopy of RA-treated EBs differentiated into neurons 2 days after plating. (b) Tubb3 labelling of RA-treated EBs differentiated into neurons 2 days after plating. (c,d). Immunoblots on extracts from differenting ES cells and EBs with the indicated antibodies. The membranes were stained with Ponceau as loading controls. (e). Immunoblots on extracts from ES cell lines engineered to re-express recombinant Taf4. (f). Phase-contrast microscopy and Tubb3 labelling of RA-treated EBs differentiated into neurons 2 days after plating. All images were taken at $\times 20$ magnification. Scale bars $100 \mu \mathrm{m}$.

WT, but not mutant EB8. The reduced activation of these genes therefore results from diminished PIC formation and elongating Pol II.

In contrast to clusters 1 and 2, where basal levels of PIC formation and Pol II occupancy were observed in ESCs, later stage activation of cluster 3 genes was associated with high levels of de novo TFIIB and Pol II recruitment and a potent increase in H3K27ac in WT EB8, but not mutant EB8 (Fig. 7e). Nevertheless, H3K4me3 was observed at these promoters in both WT and mutant ESCs suggesting that even in the absence of PIC formation they were epigenetically marked for future activation. Downregulation of genes in cluster 4 during differentiation was associated with strongly diminished TFIIB, Pol II, H3K27ac and H3K4me3 in WT EB8 showing diminshed PIC formation that was less marked in mutant cells. Consequently, Tbp was redistributed during differentiation of WT ESCs with around 14,000 sites showing preferential or unique occupancy at EB8, while occupancy of many ES sites was strongly reduced (Supplementary Fig. 9A). Tbp was enriched at the TSS of 2,086 genes mainly present in clusters 2 and 3 and strongly enriched in ontology terms associated with brain and the nervous system consistent with redistribution of Tbp from ES-expressed/pluripotency genes to those involved in neuronal differentiation (Supplementary Fig. 9B). 
a

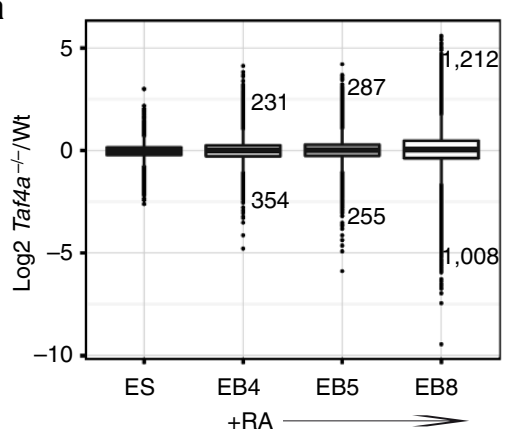

b

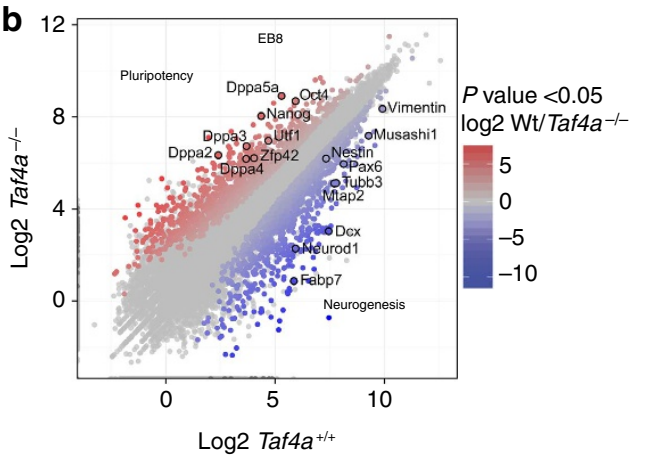

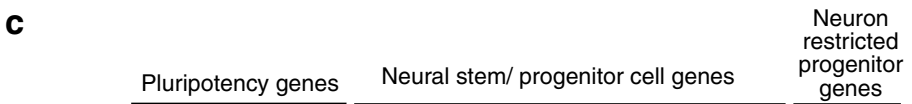

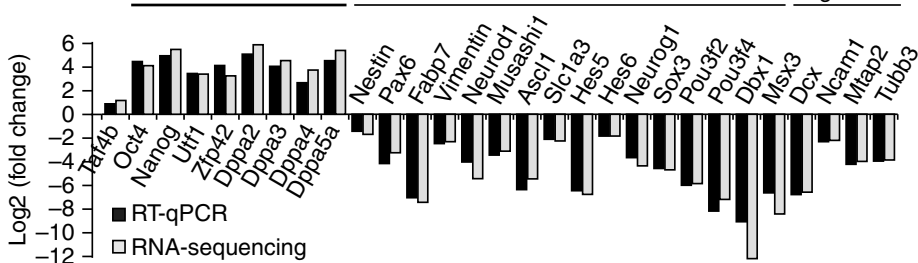

d

WT Taf4a-l-

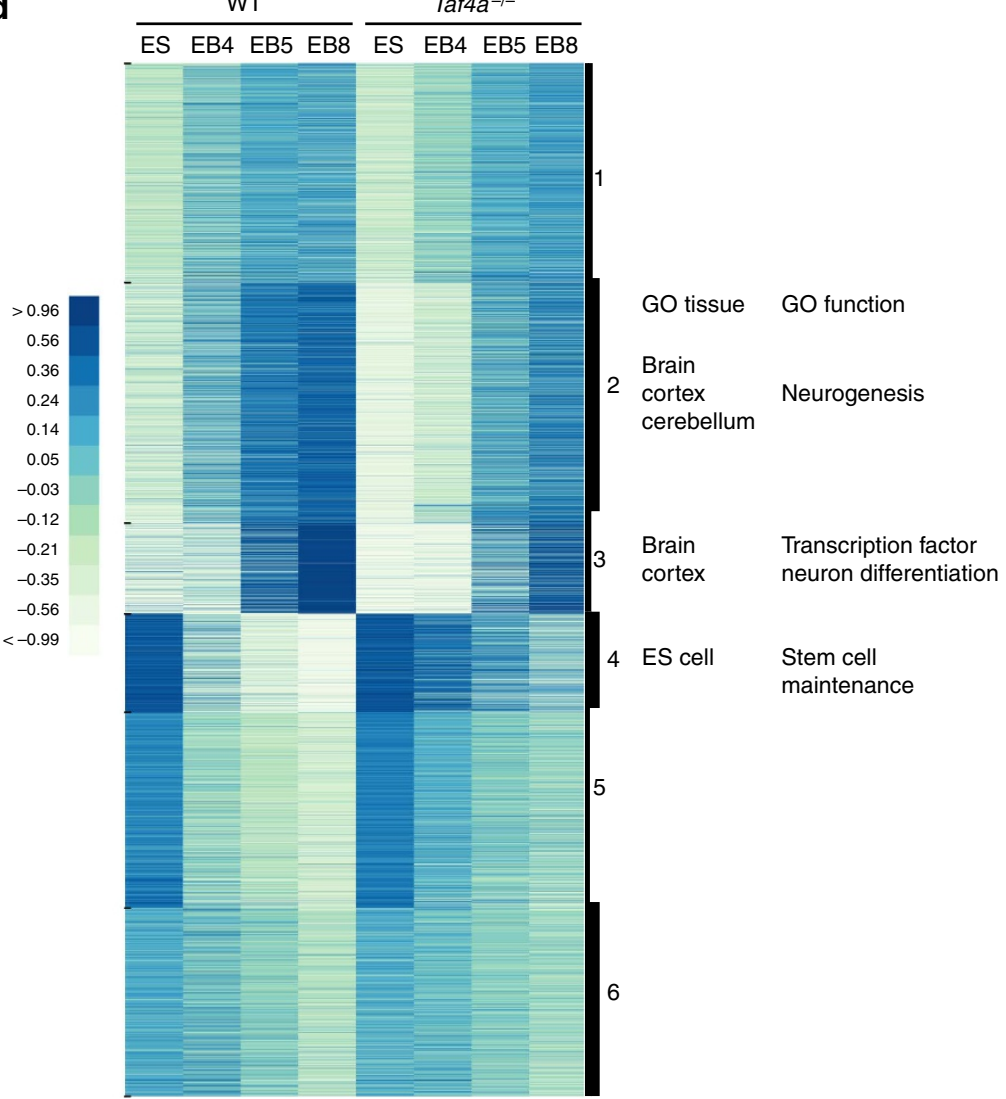

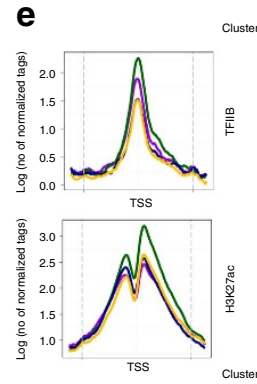
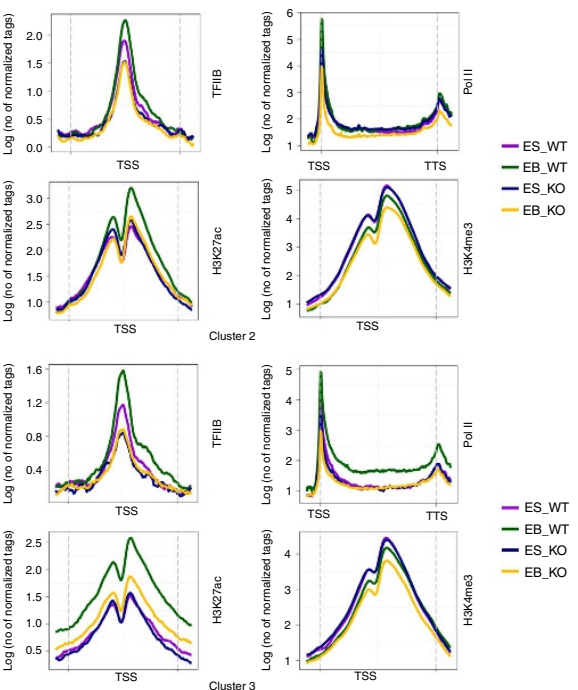

- ES_WT

- EB_WT

- ES_KO
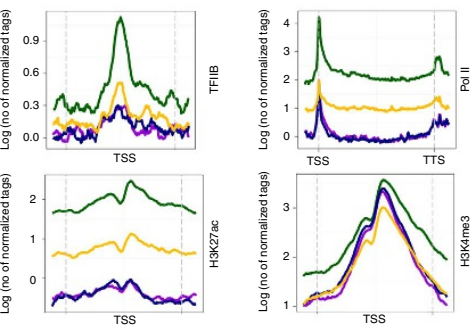

- ES_WT

- EB-WT

- EB_KO
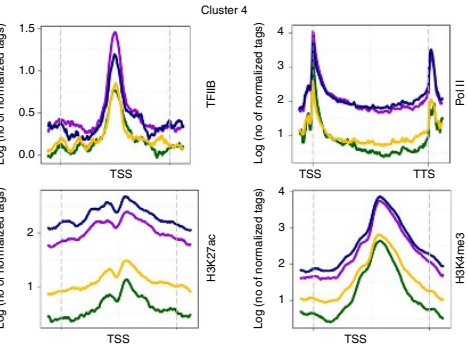

- ES_WT

- ES_KO

Figure 7 | Taf4 is required for activation of neurogenic genes in differentiating ES cells. (a). Gene expression changes at different stages of differentiation in Taf $4 a^{-/-}$compared with WT ES cells. The numbers of genes with altered expression and their fold changes at each stage are indicated. (b). Global comparison of RNA-seq data in Taf4a-/ - compared to WT at the EB8 stage. The expression of several neurogenic and pluripotency genes are highlighted. (c). Comparison of the changes in expression of selected marker genes measured by RNA-seq and by RT-qPCR. (d). Kinetics of gene expression and their alterations during differentiation are represented as a clustered heatmap. The ontology terms associated with the genes in clusters 2-4 that are most affected by Taf4 loss are indicated. (e). Metaprofiles for the indicated ChIP-seq data sets corresponding to genes in clusters 2-4 are shown. For TFIIB, H3K4me3 and H3K27ac the profiles are centred on the TSS. For Pol II the profiles are show over the gene from the transcription start site (TSS) to the transcription termination site (TTS). 
In contrast, in Taf4a-1- EB8, little occupancy of the neuronal associated WT EB8 sites was observed (Supplementary Fig. 9A). Instead, Tbp was enriched at EB8 at around 3,000 genes associated with embryos and a variety of tissues from the different germ layers (Supplementary Fig. 9C). A smaller group of around 3,000 sites appeared more strongly occupied in mutant EB8 that were associated with 400 genes with only poorly enriched ontology scores.

Together these observations are consistent with the idea that Tbp was redistributed from ES-expressed/pluripotency to neurogenic genes during differentiation of WT ESCs, whereas in Taf $4 a^{-1-}$ ESCs, Tbp redistribution was diminished, and it remained associated with ES-expressed genes. Taf4 is therefore required for de novo PIC formation necessary for activation of neurogenic genes and for efficient dissociation of the PIC at pluripotency genes.

Taf4 is essential for contractile cardiomyocyte differentiation. In the spontaneous differentiation experiments, expression of the early cardiac markers $N k \times 2-5$ and Anf was reduced and in accordance with this, we observed a lack of spontaneously contractile cardiomyocytes from the Taf $4 a^{-1}$ EBs, whereas contractile cardiomyocytes were frequently seen from WT EBs. To specifically examine cardiomyocyte differentiation, EBs were cultured for 5 days in medium containing 20\% FCS, before plating on gelatin-coated plates for a further 9 days.

Spontaneously contracting cardiomyocytes were readily identified around 3-5 days after plating of WT EBs, whereas no contractile activity of mutant cells was observed during the 9-day period. In agreement with this, Tnnt2 immunostaining revealed formation of well-defined sarcomeric structures in the differentiated WT cells, whereas such structures were severely reduced, disorganized and patchy in the Taf $4 a^{-1-}$ cells (Fig. 8a). Importantly, re-expression of full-length Taf4 restored proper Tnnt 2 expression and formation of organized and functional sarcomeric structures restoring observable contractile areas (Fig. 8b, Supplementary Fig. 10A). Taf4 is therefore essential for differentiation of contractile cardiomyocytes in vitro.

RNA-seq from day 9 differentiated WT and Taf $4 a^{-1-}$ ESCs identified 636 upregulated genes and 1,550 downregulated genes (Fig. 8c,d) associated with muscle contraction, mesoderm development, cell adhesion and signal transduction (Supplementary Fig. 10B). Expression of many early and late cardiomyocyte markers, including those involved in electrophysiology of contraction, were strongly reduced in mutant cells (Fig. 8d and Supplementary Fig. 10B). Nevertheless, as seen above, pluripotency gene expression was not properly repressed during cardiomyocyte differentiation. Taf 4 is therefore required for normal activation of the gene expression programme required for differentiation of functional cardiomyocytes.

\section{Discussion}

We define an essential role of Taf4 in mouse embryogenesis. Previous inactivation of Tbp or ubiquitous core Tafs led to preimplantation or early post-implantation embryonic death ${ }^{35-38}$. Due to this early lethality, TAF function at later stages of mammalian embryogenesis where complex lineage specification and differentiation processes occur is so far unknown. In contrast, Taf4a $a^{-1-}$ embryos survive until E9.5 and display many developmental defects. The longer survival of these embryos may be explained by redundancy with Taf $4 \mathrm{~b}$ that maintains TFIID integrity and all functions required for early development and gastrulation, after which the essential role of Taf4 in specific developmental processes can be observed. This idea is supported by the overlapping expression of Taf $4 a$ and Taf $4 b$ at early stages of development, the expression of Taf $4 b$ in the Taf $4 a^{-1-}$ embryos and more directly by our observation that Taf4 and Taf $4 \mathrm{~b}$ are redundant for ESC viability. Moreover, Taf $4 b^{-1-}$ mice are viable and show defects only at the adult stage $e^{7,39}$. Thus, while Taf4 can fully replace Taf4b during embryogenesis, Taf $4 \mathrm{~b}$ can compensate for Taf4 loss in ESCs and at early, but not later stages of embryogenesis. The observed redundancy may reflect the integration of Taf4b in TFIID in Taf $4 a^{-1-}$ ESCs to maintain its integrity. However, we cannot exclude other interpretations such as the presence of Taf $4 \mathrm{~b}$ in an alternative TFIID-like complex functioning during early development, and a requirement for Taf4-containing TFIID only at later stages to regulate expression of a distinct subset of genes that govern further development.

Taf $4 a^{-1-}$ embryos are characterized by several defects. Embryos displayed an overall atrophy resulting in part from increased apoptosis, the absence of a large trunk region and a strongly reduced forebrain domain. However, a striking feature is the absence of foregut invagination associated with lack of gut tube formation and aberrant ventral morphogenesis. In WT embryos, ventral folding morphogenesis achieves gut internalization, proper ventral localization of the heart and enclosure of the fetus in extraembryonic membranes ${ }^{40}$. Taf $4 a^{-1-}$ embryos are characterized by incomplete enclosure in the yolk sac, misplacement of the heart that develops anterior to the head and a lack of embryo turning. This aspect of the phenotype strongly resembles embryos lacking Bmp2 that arrest before turning and show many similar features in particular misplacement of the heart anterior to the head ${ }^{40}$. Analysis of Taf $4 a^{-1-}$ embryos thus confirms the importance of correct ventral morphogenesis in positioning of head and heart. Nevertheless, Taf4 is not required for the critical visceral endoderm expression of Bmp2 that persists in mutant embryos, suggesting that it may rather be required for the downstream transcriptional response of the Bmp2 pathway.

While the above phenotypes highlight processes defective in Taf $4 a^{-1-}$ embryos, others occur almost normally. Taf4 is dispensible for many patterning events, activation of early neural and mesodermal makers and the posterior development of the embryo that, in contrast to the anterior, is almost normal. Importantly, Pou5f1-expressing PGCs are formed in Taf $4 a^{-1-}$ embryos although they cannot migrate properly due to the absence of a developed hindgut. Generation of PGCs involves complex transcriptional and epigenetic reprogramming events including reactivation of pluripotency genes as well as changes in histone and DNA methylation ${ }^{41-43}$. Taf 4 is therefore not required for this naturally induced reprogramming.

This observation contrasts with a reported role of Taf4 in maintaining ESC pluripotency and promoting induced pluripotent stem cell reprogramming in vitro. Pijnappel et al. ${ }^{27}$, reported that Taf 4 overexpression enhanced the ability of Pou5f1, Nanog, Sox 2 and Myc to reprogram human and mouse fibroblasts to induced pluripotent stem cells in vitro. Furthermore, they reported that Taf4 or Taf4b short hairpin RNA knockdown resulted in spontaneous differentiation of ESCs implying that both are essential for maintaining stemness. They further proposed Taf4 as an integral component of the pluripotency network as Pou5f1 and Nanog bind a critical enhancer driving high Taf $4 a$ expression in ESCs. In contrast, Bahat et al. ${ }^{44}$ used siRNA knockdown and reported that Taf $4 \mathrm{~b}$ plays a critical role in maintaining ES cell stemness, whereas Taf4 knockdown had no effect. Thus, there are conflicting data on the role of Taf4 and Taf $4 \mathrm{~b}$ in maintaining ESC pluripotency, perhaps related to the techniques used or the efficiencies of knockdown achieved.

Our use of genetically modified mice and ESCs clearly shows that Taf4 is dispensible for pre-implantation development in vivo and that $\mathrm{Taf}_{4 a^{-1}}$ ESCs are almost indistinguishable from WT 
a
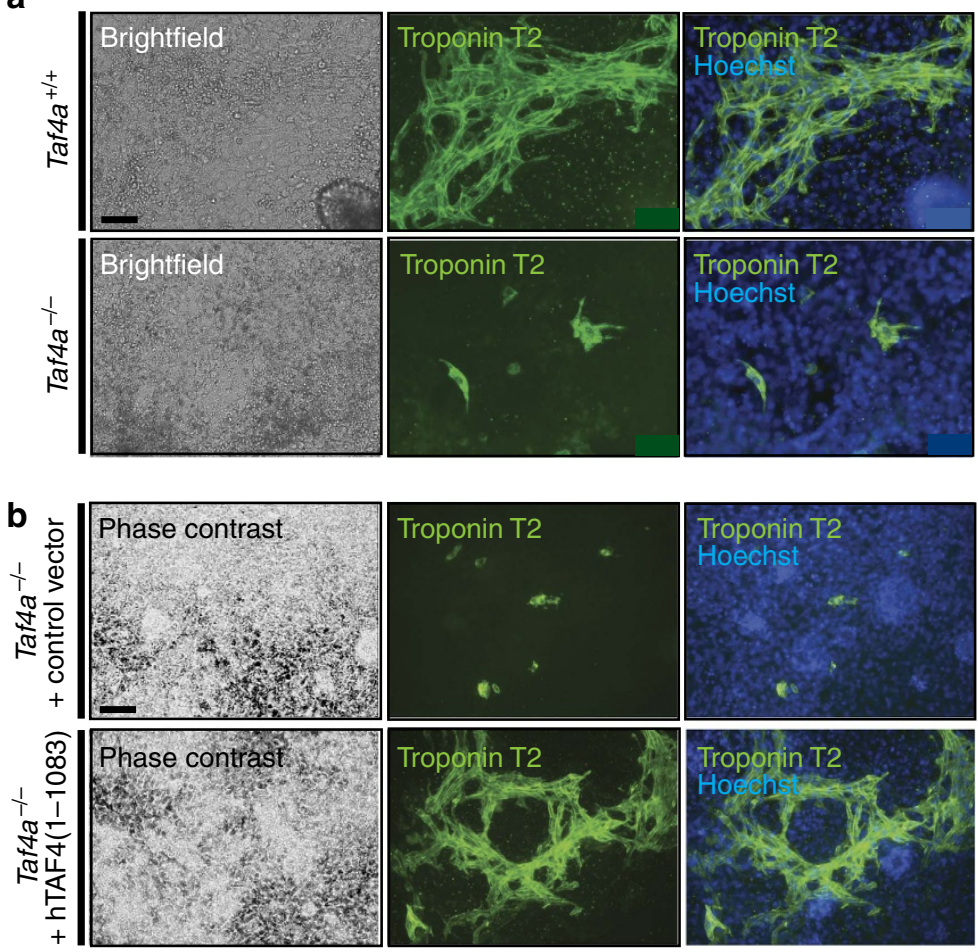

C
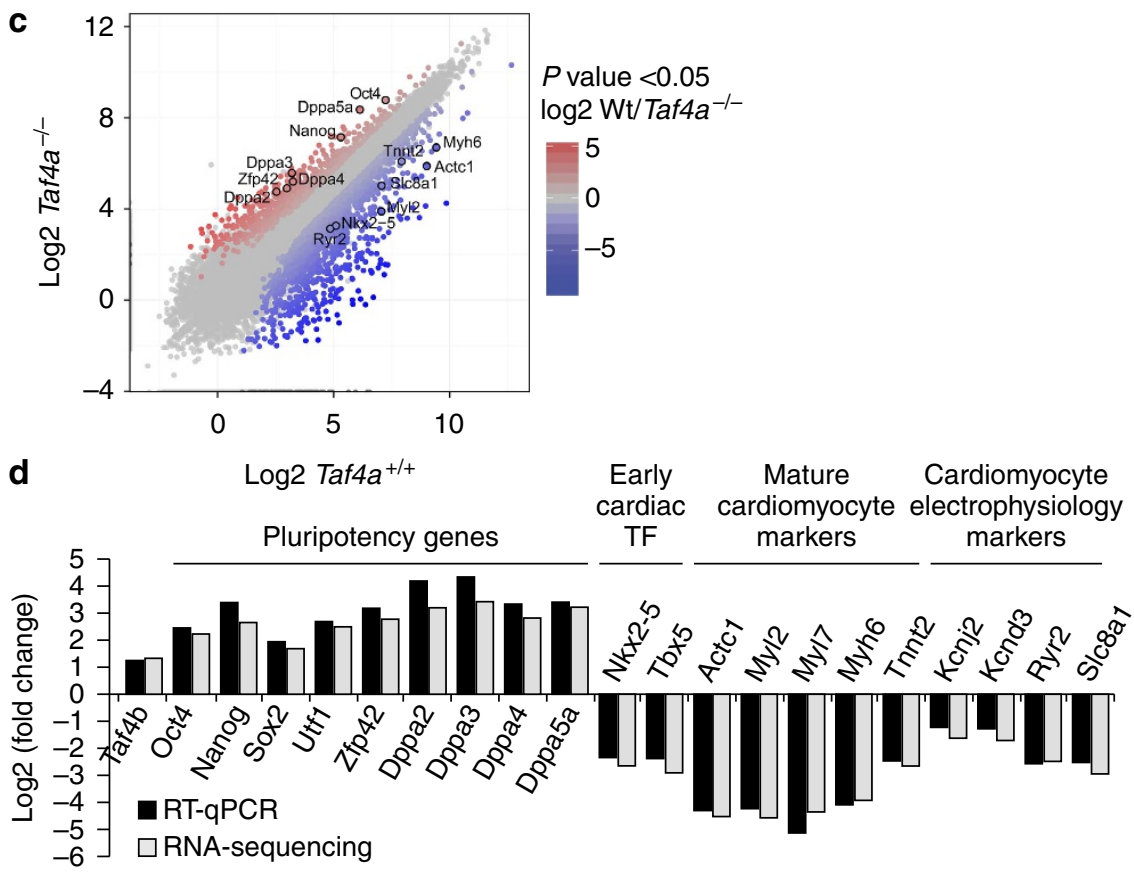

Figure 8 | Taf4 is required for cardiomyocyte differentiation. (a) Phase-contrast microscopy and Tnnt2 labelling of EBs differentiated into cardiomyocytes 9 days after plating. (b) Phase-contrast microscopy and Tnnt2 labelling of ES cells engineered to re-express exogenous Taf4 differentiated into cardiomyocytes 9 days after plating. All images were taken at $\times 20$ magnification. (c) Global comparison of RNA-seq data in Taf4a-/ - compared with WT at the EB8 stage. The expression of several cardiomyocyte markers and pluripotency genes are highlighted. (d) Comparison of the changes in expression of selected marker genes measured by RNA-seq and by RR-qPCR. Scale bars $50 \mu \mathrm{m}$.

ESCs in vitro when grown under pluripotent conditions. As Taf $4 b^{-1-}$ mice are viable, genetics indicates that neither Taf4 nor Taf $4 \mathrm{~b}$ are essential for pluripotency, but rather are redundant for this function. Moreover the appearance of PGCs in the Taf $4 a^{-1}-$ embryos indicates that Taf $4 \mathrm{~b}$ can also replace Taf4 in this reprogramming event.
Rather than playing a role in ESC pluripotency, our data show that Taf4 acts during differentiation. Similar to what is seen in vivo, Taf4a ${ }^{-1}$ EBs initiate expression of early markers of all three germ layers, but Taf4 is essential for the activation of later stage differentiation genes. Analysis of RA-driven neurogenic differentiation of Taf $4 a^{-1}$ ESCs confirmed that expression of 
early-induced genes is affected less than later markers. The activation kinetics reflect somewhat different regulatory mechanisms as promoters of early-induced genes show appreciable PIC formation in the undifferentiated state that is increased during differentiation, whereas late-induced genes show little PIC formation in ESCs, their activation being almost completely dependent on de novo PIC formation. In mutant ESCs reduced PIC formation is seen at early genes, but is not increased during differentiation, and de novo PIC formation at later genes is almost completely abolished. Thus while Taf4b-containing TFIID can assemble the PIC in ESCs, Taf4 is critical for de novo PIC formation on later stage neurogenic genes.

The defective neuronal and cardiomyocyte differentiation of Taf $4 a^{-1-}$ ESCs is reminiscent of the role of Taf3 in endodermal differentiation of ESCs ${ }^{45}$. Short hairpin RNA silencing showed that Taf3 is not required for ESC pluripotency, but plays a specific role in endoderm differentiation. Here we show that Taf4 is not required for ESC pluripotency, but plays a more general role in differentiation of all three germ layers. It is possible that the overall reduced amounts of TFIID in Taf $4 a^{-1-}$ ESCs may impact their differentiation. Pijnappel et al. ${ }^{27}$ (and Fig. 5a in this study) showed higher levels of Tbp and TAFs in ESCs compared with fibroblasts and suggested that high TFIID levels are required for pluripotency. Reduced TFIID would therefore be expected to affect pluripotency rather than differentiation. For this reason, we postulate that the defective differentiation of Taf $4 a^{-1}$ ESCs and the defects seen in Taf4null embryos reflect loss of a specific function of Taf4 rather than an overall decrease in TFIID.

Bahat et al. $^{44}$ reported that Taf4 knockdown rendered ESCs refractory to the effects of RA, whereas we show here incomplete differentiation of RA-treated Taf $4 a^{-1-}$ ESCs. However, Bahat et $a l .{ }^{44}$ treated ESCs directly with RA without prior growth as EBs, an essential feature of the neurogenic differentiation protocol. Nevertheless, an interesting feature of differentiating Taf $4 a^{-1-}$ neurons and cardiomyocytes is the persistent expression of pluripotency genes. Bahat et al. ${ }^{44}$ showed that Taf4b, but not Taf4, interacts with Pou5f1. The interactions of Pou5f1 with the Taf4b-containing TFIID in the Taf $4 a^{-1-}$ ESCs may promote expression of the pluripotency network to the detriment of redistribution to differentiation genes thereby explaining the persistent expression of pluripotency genes in absence of Taf4.

\section{Methods}

Recombinant mice. C57Bl6J mice carrying Taf4a null alleles where exons 11 and 12 have been deleted were previously described ${ }^{16}$. Taf $4 a^{-1-}$ embryos were derived from crosses of 6 to 7 -week-old $\mathrm{Taf}_{4 a^{+/-}}$animals. Mice were kept in accordance with institutional guidelines regarding the care and use of laboratory animals and in accordance with National Animal Care Guidelines (European Commission directive 86/609/CEE; French decree no.87-848). All procedures were approved by the French national ethics committee.

Histology and immunohistochemistry. Deciduae were fixed in Bouin's fluid for $14 \mathrm{~h}$ before being dehydrated and embedded in paraffin. The embedded decidua were cut in $5-\mu \mathrm{m}$ sections and stained with hematoxylin and eosin. Other sections were used for terminal deoxynucleotidyl nick-end labelling assay using the ApopTag Peroxidase In Situ.

Apoptosis Detection Kit (Millipore) as described by the manufacturer. To analyse proliferation, pregnant females were peritoneal injected with BrdU $\left(50 \mathrm{mg} \mathrm{kg}^{-1}\right.$ final) and sacrificed after $90 \mathrm{~min}$. Paraffin sections on deciduae obtained from these litters were boiled in citrate buffer for antigen retrieval and staining was performed with primary anti-BrdU (555627, BD Biosciences). Pecam staining on whole-mount embryos and yolk sacs was performed as described ${ }^{46}$.

Whole-mount in situ hybridization. Mouse embryos were dissected in ice-cold $\mathrm{PBS}$ and fixed $\mathrm{O} / \mathrm{N}$ in $4 \%$ paraformaldehyde (PFA)/PBS. After several washes in PBS- $0.1 \%$ Tween-20 (PBT) to eliminate traces of formaldehyde, embryos were bleached for $1 \mathrm{~h}$ in $3 \% \mathrm{H}_{2} \mathrm{O}_{2} / \mathrm{PBT}$ and washed $3 \times 5 \mathrm{~min}$ in PBT before being digested with Proteinase $\mathrm{K}\left(10 \mu \mathrm{g} \mathrm{ml}^{-1}\right)$ for $30 \mathrm{~s}, 1 \mathrm{~min} 30 \mathrm{~s}$ or $2 \mathrm{~min} 30 \mathrm{~s}$ (E7.5, E8.5 and E9.5). Digestion was stopped by $5 \mathrm{~min}$ incubation in $2 \mathrm{mg} \mathrm{ml}^{-1}$ glycine/ PBT. Embryos were washed again $3 \times 5 \mathrm{~min}$ in PBT before post-fixing for $20 \mathrm{~min}$ in $0.2 \%$ glutaraldehyde $/ 4 \%$ PFA/PBS. After further washes they were incubated in prewarmed hybridization buffer and prehybridized for $2 \mathrm{~h}$ at $65^{\circ} \mathrm{C}$. The buffer was then replaced with fresh prewarmed hybridization buffer containing the digoxigenin labelled RNA probes and incubated $\mathrm{O} / \mathrm{N}$ at $65^{\circ} \mathrm{C}$. The next day, embryos were washed twice in the hybridization buffer at $65^{\circ} \mathrm{C}$ before treating them with RNaseA to reduce background. The embryos were then blocked for $2 \mathrm{~h}$ in $2 \%$ blocking solution (Roche)/TBS-0.1\% Tween-20 (TBST) and incubated $\mathrm{O} / \mathrm{N}$ in the same solution containing 1:2,500 anti-digoxigenin antibody (Roche). The next day the embryos were washed in TBST, before washing them in NTMT buffer $(2 \times 10 \mathrm{~min})$ and developing the signal with BM Purple (Roche). Most in situ hybridisations were performed on three independent WT and mutant embryos.

Generation, culture and differentiation of $\mathrm{Taf4a}^{-/-}$ES cells. Taf $4 a^{+/}$mice were crossed and E3.5 blastocysts were flushed out the uterus and cultured on mitomycin treated MEF feeders. The ICM outgrowths were trypsinized and passaged on 24-well plates. ES cells were further cultured on inactivated MEF feeders under standard conditions (DMEM $4.5 \mathrm{gl}^{-1}$ glucose/Glutamax supplemented with $15 \%$ heat inactivated FCS, LIF, nonessential amino acids and $\beta$-mercaptoethanol). Taf $4 a^{-1}$ ES cells were identified by PCR as previously described ${ }^{16}$. For spontaneous differentiation, ES cells were feeder depleted and allowed to form EBs in DMEM $4.5 \mathrm{gl}^{-1}$ glucose/Glutamax supplemented with $10 \%$ heat inactivated FCS, nonessential amino acids and $\beta$-mercaptoethanol in non-adherent bacterial plates. EBs were cultured for a total of 14 days with medium change every other day. Cells were differentiated by RA into neurons as described ${ }^{33,34}$. For differentiation into cardiomyocytes, $4 \times 10^{6}$ cells were grown as EBs in EB medium containing $20 \%$ heat inactivated FCS and cultured for 5 days. EBs were then transferred to gelatin-coated plates and cultured for 9 more days. For rescue experiments, a linearized control plasmid or a plasmid containing recombinant human or mouse TAF4 complementary DNA (cDNA) was electroporated into Taf $4 a^{-I}$ ES cells and selected with puromycin. Resistant clones were further amplified and differentiated as described above.

Immunostainings and alkaline phosphatase staining. For immunostaining, cells were fixed for $30 \mathrm{~min}$ in $4 \%$ paraformaldehyde at room temperature, followed by incubation in blocking solution (PBS, $0.1 \%$ Triton X-100, $5 \%$ normal goat serum) for at least $2 \mathrm{~h}$. Primary antibodies were incubated $\mathrm{O} / \mathrm{N}$ in blocking solution and then washed 3 times with PBS, $0.1 \%$ Triton X-100 for 10 min per step to reduce unspecific binding before incubation with secondary antibodies. The Alkaline Phosphatase Detection Kit (SCR004; Millipore) was used following the manufacturers instructions.

Cell growth and cell cycle analysis. To determine growth rates, $5 \times 104$ cells from either WT or mutant ES cells, were plated gelatin-coated six-well plates in triplicate and counted for 4 consecutive days. For cell cycle analysis, cells were fixed in $70 \%$ ice-cold ethanol $\mathrm{O} / \mathrm{N}$, washed with $\mathrm{PBS}$ and then stained with propidium iodide for $4 \mathrm{~h}$. Fluorescence was detected using the BD FACSCalibur flow cytometer and the obtained data were analysed with the CellQuest software.

Gene expression analyses. Total RNA from ES cells or EBs was isolated using the GenElute Mammalian Total RNA Miniprep Kit (Sigma-Aldrich). cDNA was generated using Maxima First Strand cDNA Synthesis Kit for RT-qPCR (Thermo Scientific), following the manufacture's instructions. PCR used the SYBR Green PCR Master Mix from Qiagen and the LightCycler 480 Real-Time PCR-System (Roche). Relative expression levels were normalized to GAPDH. RNA-seq analyses were performed essentially as described ${ }^{47,48}$. All RT-qPCR experiments were performed in triplicate. Messanger RNA-seq was performed essentially as previously described ${ }^{48}$. Sequence reads mapped to reference genome mm9/NCBI37 using Tophat ${ }^{49}$. Data normalization and quantification of gene expression was performed using the DESeq 2 Bioconductor package ${ }^{50}$. Two biological replicates from two independent WT and Taf $4 a^{-1-}$ ESC lines were analysed. Gene ontology analyses were performed using the functional annotation clustering function of DAVID (http://david.abcc.ncifcrf.gov/).

Preparation of ES cell nuclear extracts, IP and immunoblot. ES cells were resuspended in swelling buffer $(25 \mathrm{mM}$ Tris $\mathrm{pH} 7,9,10 \mathrm{mM} \mathrm{KCl}, 1.5 \mathrm{mM} \mathrm{MgCl} 2$, $1 \mathrm{mM}$ DTT), incubated for $10 \mathrm{~min}$ on ice, lysed using a dounce homogenizer and centrifuged. Pelleted nuclei were resuspended in extraction buffer $(50 \mathrm{mM}$ Tris $\mathrm{pH}$ 7,5, $400 \mathrm{mM} \mathrm{NaCl}, 0.5 \% \mathrm{NP}-40,5 \%$ Glycerol and $1 \times$ Roche complete protease inhibitor) and proteins were extracted for $2 \mathrm{~h}$ at $4{ }^{\circ} \mathrm{C}$ with rotation. For IP, $500 \mu \mathrm{g}$ nuclear extract were pre-cleared for $2 \mathrm{~h}$ with $50 \mu \mathrm{l}$ of protein $\mathrm{G}$ sepharose beads, before addition of with $1 \mu \mathrm{g}$ of anti-TBP (3G3) antibody $\mathrm{O} / \mathrm{N}$ at $4^{\circ} \mathrm{C}$. The next day, bound complexes were recovered by incubating the extract with $25 \mu$ protein $\mathrm{G}$ sepharose beads for $2 \mathrm{~h}$ at $4^{\circ} \mathrm{C}$. Beads containing bound proteins were washed three times with extraction buffer for $15 \mathrm{~min}$ per wash and once with the buffer containing $150 \mathrm{mM} \mathrm{NaCl}$ before being boiled in in SDS loading buffer for $5 \mathrm{~min}$ at 
$95^{\circ} \mathrm{C}$. Bound proteins were analysed by Western blot using the indicated antibodies. A control IP for each extract was performed in parallel using a non-specific antibody against glutathione-S transferase (GST). Uncropped western blots are provided for all immunoblots in Supplementary Figure 11.

Gel filtration. $500 \mu \mathrm{l}$ of nuclear extracts prepared as described above containing $6 \mathrm{mg}$ of protein were injected onto a equilibrated Superose $6(10 / 300)$ column and run at $0.4 \mathrm{ml} \mathrm{min}^{-1} .500 \mu \mathrm{l}$ fractions were collected and analysed by western blot.

Antibodies. The following antibodies were used: anti-OCT4 (sc-9081; Santa Cruz), anti-NANOG (RCAB001P; Reprocell), SOX2 (L1D6A2; Cell Signaling), previously described or recently developed in house antibodies against TBP (3G3), TAF1, TAF3 (ref. 15), TAF4 (32TA and also sc-136093; Santa Cruz), TAF4B (ref. 16), TAF5 (1TA), TAF6 (25TA), TAF7 (19TA), TAF12 (22TA), and TAF13 (16TA), TAF10 (sc-102125; Santa Cruz) and in house 6TA2B11 (ref. 51), anti-Pol II (sc-9001x; Santa Cruz), anti-Tnnt2 (MS-295-P1; Thermo scientific). We also used a polyclonal TAF4B antibody (2057) raised against a peptide corresponding to amino acids $692-710$ of human TAF4B that is $100 \%$ conserved in mouse Taf4b. This antibody also recognizes Taf4 as this region comprises only two amino acid differences with Taf $4 \mathrm{~b}$. The following antibody dilutions were used for immunostaining. Pou5f1 and Nanog, 1:100; Sox2, and Taf4, 1:50; Tubb3 and Tnnt2, 1:1,000. For immunoblots all antibodies were diluted 1:1,000 except polyclonal serum 2057 that was diluted 1:2,000.

Chromatin IP sequencing. Chromatin IP experiments were performed on $0,4 \%$ PFA-fixed chromatin according to standard protocols as previously described ${ }^{15}$. Chromatin IP sequencing libraries were prepared and sequenced on an Illumina Hi-seq2500 as single-end 50-base reads. After sequencing, peak detection was performed using the MACS software (ref. $52 \mathrm{http} / / /$ liulab.dfci. harvard.edu/MACS/). Global clustering, meta analyses and quantitative comparisons were performed using seqMINER ${ }^{53}$ and R (http://www.r- project.org/).

\section{References}

1. Wright, K. J., Marr, 2nd M. T. \& Tjian, R. TAF4 nucleates a core subcomplex of TFIID and mediates activated transcription from a TATA-less promoter. Proc. Natl Acad. Sci. USA 103, 12347-12352 (2006).

2. Cler, E., Papai, G., Schultz, P. \& Davidson, I. Recent advances in understanding the structure and function of general transcription factor TFIID. Cell Mol. Life Sci. 66, 2123-2134 (2009).

3. Bieniossek, C. et al. The architecture of human general transcription factor TFIID core complex. Nature 493, 699-702 (2013).

4. Zhou, H. et al. Dual functions of TAF7L in adipocyte differentiation. eLife 2, e00170 (2013).

5. Cheng, Y. et al. Abnormal sperm in mice lacking the taf7l gene. Mol. Cell. Biol. 27, 2582-2589 (2007).

6. Zhou, H., Wan, B., Grubisic, I., Kaplan, T. \& Tjian, R. TAF7L modulates brown adipose tissue formation. Elife 3, e02811 (2014)

7. Falender, A. E. et al. Maintenance of spermatogenesis requires TAF4b, a gonad-specific subunit of TFIID. Genes Dev. 19, 794-803 (2005).

8. Voronina, E. et al. Ovarian granulosa cell survival and proliferation requires the gonad-selective TFIID subunit TAF4b. Dev Biol. 303, 715-726 (2007).

9. Herrera, F. J., Yamaguchi, T., Roelink, H. \& Tjian, R. Core promoter factor TAF9B regulates neuronal gene expression. Elife 3, e02559 (2014).

10. Martianov, I. et al. Late arrest of spermiogenesis and germ cell apoptosis in mice lacking the TBP-like TLF/TRF2 gene. Mol. Cell. 7, 509-515 (2001).

11. Gazdag, E. et al. TBP2 is essential for germ cell development by regulating transcription and chromatin condensation in the oocyte. Genes Dev. 23, 2210-2223 (2009).

12. Gangloff, Y., Romier, C., Thuault, S., Werten, S. \& Davidson, I. The histone fold is a key structural motif of transcription factor TFIID. Trends Biochem. Sci. 26, 250-257 (2001).

13. Werten, S. et al. Crystal structure of a subcomplex of human transcription factor TFIID formed by TATA binding protein-associated factors hTAF4 (hTAF(II)135) and hTAF12 (hTAF(II)20. J. Biol. Chem. 277, 45502-45509 (2002).

14. Alpern, D. et al. TAF4, a subunit of transcription factor II D, directs promoter occupancy of nuclear receptor HNF4A during post-natal hepatocyte differentiation. Elife 3, e03613 (2014).

15. Thuault, S. et al. Functional analysis of the TFIID-specific yeast TAF4 (yTAF(II)48) reveals an unexpected organization of its histone-fold domain. J. Biol. Chem. 277, 45510-45517 (2002).

16. Mengus, G. et al. TAF4 inactivation in embryonic fibroblasts activates TGFbeta signalling and autocrine growth. EMBO J. 24, 2753-2767 (2005).

17. Fadloun, A. et al. The TFIID subunit TAF4 regulates keratinocyte proliferation and has cell-autonomous and non-cell-autonomous tumour suppressor activity in mouse epidermis. Development 134, 2947-2958 (2007).
18. Fadloun, A. et al. Retinoic acid induces TGFbeta-dependent autocrine fibroblast growth. Oncogene 27, 477-489 (2008).

19. Martianov, I. et al. TAF4 inactivation reveals the 3 dimensional growth promoting activities of collagen 6A3. PLoS ONE 9, e87365 (2014).

20. Wilt, F. H. Erythropoiesis in the chick embryo: the role of endoderm. Science 147, 1588-1590 (1965).

21. Miura, Y. \& Wilt, F. H. Tissue interaction and the formation of the first erythroblasts of the chick embryo. Dev. Biol. 19, 201-211 (1969).

22. Palis, J., McGrath, K. E. \& Kingsley, P. D. Initiation of hematopoiesis and vasculogenesis in murine yolk sac explants. Blood 86, 156-163 (1995).

23. Kaufman. The Atlas of Mouse Development (Academic Press, 1998).

24. Perea-Gomez, A., Rhinn, M. \& Ang, S. L. Role of the anterior visceral endoderm in restricting posterior signals in the mouse embryo. Int. J. Dev. Biol. 45, 311-320 (2001).

25. Rhinn, M. et al. Sequential roles for Otx2 in visceral endoderm and neuroectoderm for forebrain and midbrain induction and specification. Development 125, 845-856 (1998).

26. Ang, S. L. et al. A targeted mouse Otx2 mutation leads to severe defects in gastrulation and formation of axial mesoderm and to deletion of rostral brain. Development 122, 243-252 (1996).

27. Pijnappel, W. W. et al. A central role for TFIID in the pluripotent transcription circuitry. Nature 495, 516-519 (2013).

28. Richardson, B. E. \& Lehmann, R. Mechanisms guiding primordial germ cell migration: strategies from different organisms. Nat. Rev. Mol. Cell Biol. 11, 37-49 (2010).

29. Tam, P. P. \& Snow, M. H. Proliferation and migration of primordial germ cells during compensatory growth in mouse embryos. J. Embryol. Exp. Morphol. 64 , 133-147 (1981).

30. Ginsburg, M., Snow, M. H. \& McLaren, A. Primordial germ cells in the mouse embryo during gastrulation. Development 110, 521-528 (1990).

31. Maston, G. A. et al. Non-canonical TAF complexes regulate active promoters in human embryonic stem cells. Elife 1, e00068 (2012).

32. Doetschman, T. C., Eistetter, H., Katz, M., Schmidt, W. \& Kemler, R. The in vitro development of blastocyst-derived embryonic stem cell lines: formation of visceral yolk sac, blood islands and myocardium. J. Embryol. Exp. Morphol. 87, 27-45 (1985).

33. Bibel, M. et al. Differentiation of mouse embryonic stem cells into a defined neuronal lineage. Nat. Neurosci. 7, 1003-1009 (2004).

34. Bibel, M., Richter, J., Lacroix, E. \& Barde, Y. A. Generation of a defined and uniform population of CNS progenitors and neurons from mouse embryonic stem cells. Nat. Protoc. 2, 1034-1043 (2007).

35. Martianov, I., Viville, S. \& Davidson, I. RNA polymerase II transcription in murine cells lacking the TATA binding protein. Science 298, 1036-1039 (2002)

36. Mohan, I. W., Scheer, E., Wendling, O., Metzger, D. \& Tora, L. TAF10 (TAF(II) 30) is necessary for TFIID stability and early embryogenesis in mice. Mol. Cell. Biol. 23, 4307-4318 (2003).

37. Gegonne, A. et al. The general transcription factor TAF7 is essential for embryonic development but not essential for the survival or differentiation of mature T cells. Mol. Cell. Biol. 32, 1984-1997 (2012).

38. Voss, A. K. et al. Taube nuss is a novel gene essential for the survival of pluripotent cells of early mouse embryos. Development 127, 5449-5461 (2000).

39. Falender, A. E., Shimada, M., Lo, Y. K. \& Richards, J. S. TAF4b, a TBP associated factor, is required for oocyte development and function. Dev. Biol. 288, 405-419 (2005).

40. Madabhushi, M. \& Lacy, E. Anterior visceral endoderm directs ventral morphogenesis and placement of head and heart via BMP2 expression. Dev. Cell 21, 907-919 (2011).

41. Messerschmidt, D. M., Knowles, B. B. \& Solter, D. DNA methylation dynamics during epigenetic reprogramming in the germline and preimplantation embryos. Genes Dev. 28, 812-828 (2014).

42. Saitou, M. \& Yamaji, M. Primordial germ cells in mice. Cold Spring Harb. Perspect. Biol. 4 (2012).

43. Saitou, M., Kagiwada, S. \& Kurimoto, K. Epigenetic reprogramming in mouse pre-implantation development and primordial germ cells. Development 139, 15-31 (2012).

44. Bahat, A. et al. TAF4b and TAF4 differentially regulate mouse embryonic stem cells maintenance and proliferation. Genes Cells 18, 225-237 (2013).

45. Liu, Z., Scannell, D. R., Eisen, M. B. \& Tjian, R. Control of embryonic stem cell lineage commitment by core promoter factor, TAF3. Cell 146, 720-731 (2011).

46. Schlaeger, T. M., Qin, Y., Fujiwara, Y., Magram, J. \& Sato, T. N. Vascular endothelial cell lineage-specific promoter in transgenic mice. Development 121, 1089-1098 (1995).

47. Laurette, P. et al. Transcription factor MITF and remodeller BRG1 define chromatin organisation at regulatory elements in melanoma cells. eLife 4, doi: 10.7554/eLife.06857 (2015).

48. Herquel, B. et al. Trim24-repressed VL30 retrotransposons regulate gene expression by producing noncoding RNA. Nat Struct. Mol. Biol. 20, 339-346 (2013). 
49. Trapnell, C., Pachter, L. \& Salzberg, S. L. TopHat: discovering splice junctions with RNA-Seq. Bioinformatics 25, 1105-1111 (2009).

50. Anders, S. \& Huber, W. Differential expression analysis for sequence count data. Genome Biol. 11, R106 (2010).

51. Indra, A. K. et al. TAF10 is required for the establishment of skin barrier function in foetal, but not in adult mouse epidermis. Dev. Biol. 285, 28-37 (2005).

52. Zhang, Y. et al. Model-based analysis of ChIP-Seq (MACS). Genome Biol. 9, R137 (2008).

53. Ye, T. et al. seqMINER: an integrated ChIP-seq data interpretation platform. Nucleic Acids Res. 39, e35 (2011).

\section{Acknowledgements}

We thank L. Tora for antibodies against TAF1 and TAF10, S. Viville for human ESC extracts and helpful discussions, all the staff of the IGBMC high throughput sequencing facility, a member of 'France Génomique' consortium (ANR10-INBS-09-08). This work was supported by grants from the CNRS, the INSERM, the Ligue Nationale et Départementale contre le Cancer (Région Alsace), the Institut National du Cancer (INCa), the ANR-10-LABX-0030-INRT French state fund through the ANR under the programme Investissements d'Avenir labelled ANR-10-IDEX-0002-02. ID is 'équipe labellisée' of the Ligue Nationale contre le Cancer. The funders had no role in study design, data collection and analysis, decision to publish, or preparation of the manuscript.

\section{Author contributions}

D.L. performed all embryo analyses in collaboration with M.R and P.D. D.L also performed analyses and differentiation of ES cells and analysed the RNA-seq with C.K.,
D.A. and I.M. performed and analysed the ChIP-seq data. G.M. generated and managed the recombinant mice, isolated the ES cell lines and performed embryo dissections I.D. D.L., M.R., P.D. and G.M. conceived experiments, analysed data and wrote the paper.

\section{Additional information}

Accession code: The RNA-seq data have been deposited in the GEO database under accession code GSE70726 and the ChIP-seq data under the accession code GSE70661. Supplementary Information accompanies this paper at http://www.nature.com/ naturecommunications

Competing financial interests: The authors declare no competing financial interests.

Reprints and permission information is available online at http://npg.nature.com/ reprintsandpermissions/

How to cite this article: Langer, D. et al. Essential role of the TFIID subunit TAF4 in murine embryogenesis and embryonic stem cell differentiation. Nat. Commun. 7:11063 doi: $10.1038 /$ ncomms11063 (2016).

\section{(c) (1)}

This work is licensed under a Creative Commons Attribution 4.0 International License. The images or other third party material in this article are included in the article's Creative Commons license, unless indicated otherwise in the credit line; if the material is not included under the Creative Commons license, users will need to obtain permission from the license holder to reproduce the material. To view a copy of this license, visit http://creativecommons.org/licenses/by/4.0/ 